\title{
Lorentzian geometry and variability reduction in airplane boarding: Slow passengers first outperforms random boarding
}

\author{
Sveinung Erland (1)* \\ Department of Maritime Studies, Western Norway University of Applied Sciences, N-5528 Haugesund, Norway
}

Jevgenijs Kaupužs $\mathbb{P}$

Faculty of Materials Science and Applied Chemistry, Institute of Technical Physics, Riga Technical University, LV-1048 Riga, Latvia and Institute of Mathematical Sciences and Information Technologies, University of Liepaja, LV-3401 Liepaja, Latvia

\author{
Albert Steiner (1) \\ Institute of Data Analysis and Process Design, Zurich University of Applied Sciences ZHAW, 8401 Winterthur, Switzerland \\ Eitan Bachmat (1) \\ Department of Computer Science, Ben-Gurion University, Beer-Sheva 84105, Israel
}

(Received 5 February 2021; accepted 7 May 2021; published 28 June 2021)

\begin{abstract}
Airlines use different boarding policies to organize the queue of passengers waiting to enter the airplane. We analyze three policies in the many-passenger limit by a geometric representation of the queue position and row designation of each passenger and apply a Lorentzian metric to calculate the total boarding time. The boarding time is governed by the time each passenger needs to clear the aisle, and the added time is determined by the aisle-clearing time distribution through an effective aisle-clearing time parameter. The nonorganized queues under the common random boarding policy are characterized by large effective aisle-clearing time. We show that, subject to a mathematical assumption which we have verified by extensive numerical computations in all realistic cases, the average total boarding time is always reduced when slow passengers are separated from faster passengers and the slow group is allowed to enter the airplane first. This is a universal result that holds for any combination of the three main governing parameters: the ratio between effective aisle-clearing times of the fast and the slow groups, the fraction of slow passengers, and the congestion of passengers in the aisle. Separation into groups based on aisle-clearing time allows for more synchronized seating, but the result is nontrivial, as the similar fast-first policy-where the two groups enter the airplane in reverse order-is inferior to random boarding for a range of parameter settings. The asymptotic results conform well with discrete-event simulations with realistic numbers of passengers. Parameters based on empirical data, with hand luggage as criteria for separating passengers into the slow and fast groups, give an $8 \%$ reduction in total boarding time for slow first compared to random boarding.
\end{abstract}

DOI: 10.1103/PhysRevE.103.062310

\section{INTRODUCTION}

Determination and optimization of the macroscopic properties of complex systems are of importance in many fields of physics. In airplane boarding, the main observable is the boarding time, which is the time it takes to get all passengers seated. The boarding time is affected by several factors, including sequencing of the queue, passenger interactions, and the time each passenger needs to clear the aisle. Moreover,

\footnotetext{
*Corresponding author: sver@hvl.no

Published by the American Physical Society under the terms of the Creative Commons Attribution 4.0 International license. Further distribution of this work must maintain attribution to the author(s) and the published article's title, journal citation, and DOI.
}

the dynamics of the passenger queue become increasingly complex as the number of passengers $N$ increases.

While the quantification of properties of complex systems often requires extensive simulations, the airplane boarding process can be analyzed in terms of a geometrical representation of the queue position and the row designation of the passengers. When the number of passengers $N \rightarrow \infty$, a flat Lorentzian metric enables the boarding time to be expressed in analytical terms, which also enables rough estimates for finite numbers of passengers [1,2]. The boarding time is found to be of leading order $\sqrt{N}$, and it is scaled by a prefactor that is governed by three main parameters (that will be explained later). The analytical expressions enable a direct optimization over these parameters [3-9].

In terms of statistical physics, airplane boarding can be viewed as a particle process where the passengers constitute an ensemble of interacting particles of which the macroscopic observables are of interest [10]. The geometric representation, 
consisting of a simple two-dimensional diagram, is directly linked to space-time geometry. Our approach is also directly related to the causal set program of quantum gravity [11-15]. In terms of the causal set program, the main contribution of this work is that we consider scenarios where each event has a stochastic proper time contribution.

When the aisle-clearing time $X$ of each passenger is stochastic, there exists a constant $\tau_{X}$ such that the asymptotic boarding time $T$ is the same as if the aisle-clearing time was constant for all passengers with value $X=\tau_{X}$. We therefore call $\tau_{X}$ the effective aisle-clearing time. The parameter is another macroscopic property of airplane boarding, and it is similar to what in material science is described as effective transport and optical properties for composite materials [16-19]. As in material sciences, the computation of $\tau_{X}$ is not trivial, and one of the main contributions of this work is to devise a method for precise estimation of the parameter. We utilize the property that $\tau_{X}$ depends on the aisle-clearing time distribution only, and interestingly this also reduces the task to the mathematical problem of finding the heaviest increasing subsequence in a permutation [20].

We study the airplane boarding process from the time that passengers have lined up in a queue outside the airplane until the last passengers are seated at their reserved seats. Empirical data shows that a reduction of the boarding time would reduce the total turnaround time and hence reduce airline costs [21]. Still, most passengers have experienced that boarding is a seemingly chaotic process where most of the time is spent in the queue waiting.

The way airlines organize the queue prior to boarding is called a boarding policy. The random boarding policy, with its completely unorganized queues, is surprisingly common, in particular for intracontinental flights in Europe [22]. There have been several efforts to find optimal policies [23], but airlines hesitate to apply the solutions since optimal queues require that individual passengers adhere to specific positions in the queue [24-27], and such detailed regulation could be detrimental to passenger satisfaction. Aspects such as priority boarding of passengers with high revenue tickets will also limit the potential for optimization.

Many airlines do enforce boarding policies with weaker restrictions, where passengers typically are divided into two or more groups. A common policy is the back-to-front policy where the first group to enter the queue is passengers with designated seats in the back of the airplane. The next group will be seated at consecutive rows closer to the front, and so on. In spite of these efforts, the policy tends to increase boarding time compared to random boarding [6]. A less common policy is the window-middle-aisle policy where groups designated to window seats are asked to sit first, followed by the middle seats and so on. However, here passengers that travel together run the risk of getting separated during boarding.

Other applied policies involve priority boarding where, e.g., groups consisting of small children or other needing assistance are asked to enter first. This is actually a variant of the slow-first policy where passengers who are expected to use a long time to clear the aisle (the slow group) are allowed to enter first, followed by the faster passengers. The universal result in Ref. [9] states that the slow-first policy is always superior to the opposite fast-first policy. In this paper, we prove another universal result, namely that slow first is also universally better than the random boarding policy. The result is a confirmation of the simulation-based result in Ref. [28]. We quantify the result by taking parameters from empirical data where the slow group is defined to be passengers with overhead bin luggage. Other boarding policy models that take the aisle-clearing time into account exist, but even though they have the potential to reduce the boarding time even further, they all assign specific position in the queue for each passenger [29-32]. As noted above, this is most likely not beneficial for customer satisfaction.

The structure of the paper is as follows. In Sec. II, we describe the boarding process, followed by a summary of the main results in Sec. III. Main parameters of the boarding process, its geometric representation, and the blocking chains that lead to the asymptotic boarding time in the many-passenger limit $(N \rightarrow \infty)$ are presented in Sec. IV. In Sec. V, we present bounds for the effective aisle-clearing time $\tau_{X}$ that is needed for computing the asymptotic boarding time when the aisleclearing time $X$ varies between passengers within each group. $\tau_{X}$ is particularly important for random boarding, and we prescribe an algorithm that enables precise estimation of $\tau_{X}$ and argue that $\sqrt{\left\langle X^{2}\right\rangle}$ serves as a lower bound for $\tau_{X}$. In Sec. VI, we present and compare analytical results for the asymptotic boarding time of the slow-first (SF), the fast-first (FF), and the random boarding (RA) policies. We also demonstrate by simulations that the large- $N$ limit results hold for realistic numbers of passengers. We end the paper with a discussion in Sec. VII.

\section{THE BOARDING PROCESS}

We consider the boarding process from the time the passengers stand in line right outside the airplane until the last passenger is seated. Once the passengers have entered the airplane, we assume that the queue order is maintained throughout the process and that a passenger in front cannot be passed in the aisle until that passenger has cleared the aisle and is seated. This means that most of the time the passengers stand still in the queue waiting for other passengers in front to take their seat.

The boarding is modeled as an iterative, two-step process: First, passengers move as far as they can get toward their designated row but must stop short in the aisle if they are blocked by other passengers. This is assumed to take a negligible amount of time compared to the next step, where passengers who have arrived at their designated row use a certain aisle-clearing time to organize luggage and take a seat.

This is illustrated by a simple example in Fig. 1, consisting of $N=8$ passengers, all having the same aisle-clearing time. At each time step, the queue moves forward and some passengers are able to sit down. However, most passengers are delayed by other passengers blocking the aisle. For example, at $t=1$ the second passenger in the queue aiming for row 4 must wait for the passenger sitting down at row 3 since the latter has a lower row number. Other passengers are blocked by displacement: The fourth passenger in the queue heading for row 2 does not have to pass any other passenger but must still wait for passengers in front to proceed. If there were more room for passengers to stand closer in the aisle (less 
(a) $t=1$

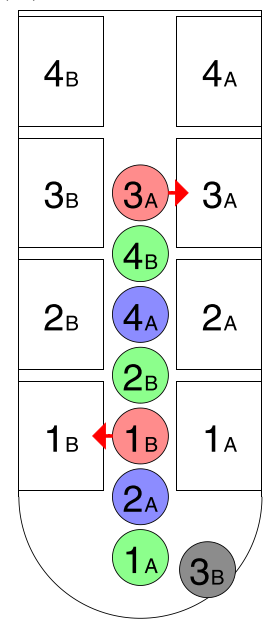

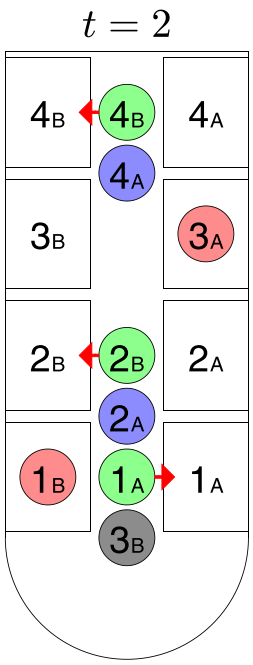

(b)

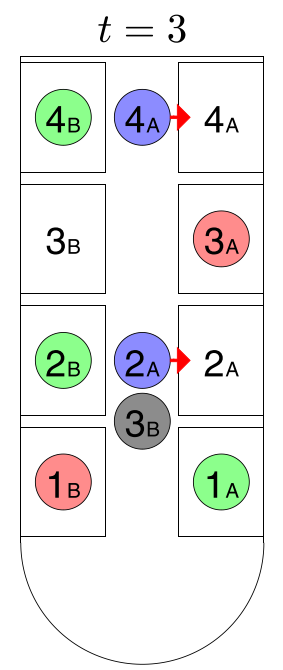

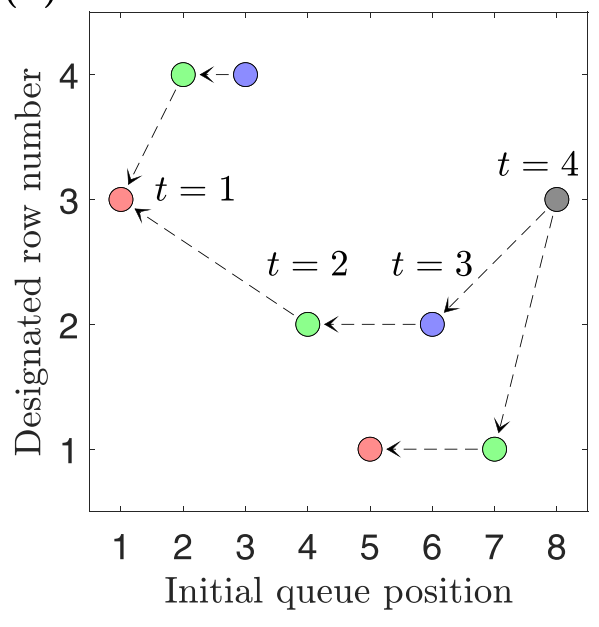

FIG. 1. (a) Boarding process shown as the stepwise advance of a queue, with $N=8$ passengers, four rows and two seats per row. Each circle represents a passenger with designated row number. At each time step, the queue moves forward, and passengers who have arrived at their designated rows sit down simultaneously. Passengers that take their seat at that time step are marked by red arrows, and each group of passengers that sit down simultaneously is color coded. (b) Each point in the queue-row diagram represents a passenger, indicated by initial queue position and designated row number. The color codes highlight groups of passengers that sit down simultaneously. Each passenger in a group has been blocked by at least one passenger in a preceding group, some of them indicated by arrows. Passengers that consecutively block each other form blocking chains, and the sum of aisle-clearing times of the passengers in the maximal blocking chain determines the boarding time, $T=4$.

congestion), the displacement effect would be less pronounced. The boarding time $T$ is the time until the last passenger is seated, and in Fig. 1, $T=4$ time steps.

The $q r$ diagram in Fig. 1(b) conveys each passenger's initial queue position $q$ and designated row number $r$ in the airplane. Groups of passengers that sit down simultaneously are color coded. The boarding time can be obtained by summing up the time it takes for each group of passengers to clear the aisle.

An alternative strategy, which is used in this paper, is to utilize the property that each person in a group has been blocked by at least one passenger in the preceding group. The $q r$ diagram is used as a tool to analyze the entire hierarchy of blocking between passengers for a given queue and to find sequences (chains) of passengers that consecutively block each other according to a blocking relation [9]. The boarding time can then be found by considering the blocking chain that needs the most time to seat all its passengers. In Fig. 1(b) three different blocking chains are shown, and the longest one consists of four passengers that have to be seated one after the other. The boarding time is the sum of those passengers' aisle-clearing times.

\section{MAIN RESULTS}

The boarding process is characterized by three key parameters. The congestion $k$ is the ratio between the length of the initial queue to the aisle length, typically in the range of three to five for common passenger airplanes (see Sec. IV A for a more detailed description). The second parameter is the fraction $p$ of passengers with long aisle-clearing time, i.e., passengers who are considered slow. The remaining fraction $(1-p)$ are fast passengers. Both the slow and fast groups have their own aisle-clearing time distribution, and each distribution is characterized by an inherent effective aisleclearing time parameter. The ratio $C$ of the effective aisleclearing time of the fast group to the effective aisle-clearing time of the slow group is the third parameter.

The main results of this paper are illustrated in Fig. 2. The total boarding time of the slow-first and fast-first policies are compared to the random boarding policy for increasing congestion $k$. In the subsequent sections, we prove rigorously the universal nature of the main features in Fig. 2 and that they apply for any set of parameters.

In Fig. 2, the congestion $k$ is on the horizontal axis, the fraction of slow passengers is $p=10 \%$, and the effective aisle-clearing time of the slow passengers is twice as long as for the fast passengers, i.e., $C=0.5$. The average total boarding time for the slow-first policy is less than for the random boarding policy for all values of $k$, both for finite numbers of passengers $N=180$ (red bullets) and $N \rightarrow \infty$ (red, dashed curve). However, the quite similar fast-first policy (blue curves) does not show this universal feature, as there is a range around $k \approx 1$ where fast first is inferior to random boarding. This highlights that the universal result for slow first is highly nontrivial.

When $N=180$, all three policies approach the same values as $k \rightarrow \infty$, since then each passenger would fill up the whole aisle. The seemingly erratic behavior for $k \approx 10$ is due to sudden changes when $N=180$ is a multiple of $k$.

The total boarding time in Fig. 2 can be determined by what is called the heaviest chain of passengers. This is illustrated by the $q r$ diagrams in Fig. 3. A passenger cannot sit down before all preceding passengers in the chain are seated. The weight of a chain is the sum of the aisle-clearing times (weights) for passengers that belong to the that chain. The boarding time is given by the weight of the heaviest chain, and that chain follows the asymptotic heaviest curve (the geodesic), with 


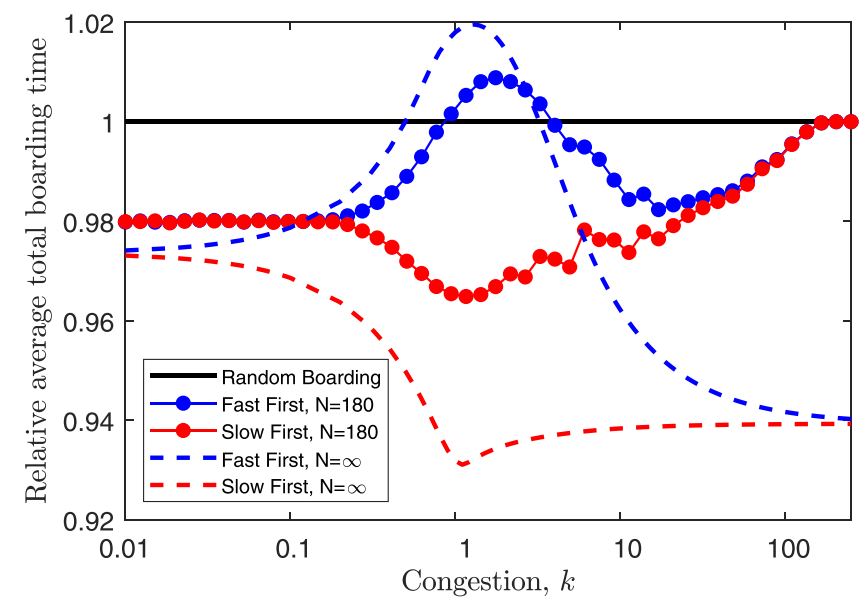

FIG. 2. Total boarding time of the slow-first (red curves) and the fast-first (blue curves) policies relative to the random boarding policy (black curve) for increasing passenger congestion $k$. We used realistic parameters with $10 \%$ slow passengers $(p=0.1)$ and assumed that the fast passengers (e.g., those with little hand luggage) clear the aisle twice as fast as the slow passengers $(C=0.5)$. We assumed there are six seats per row, and a total of $N=180$ passengers. The results are similar for the asymptotic case with an infinite number of passengers ( $N=\infty$, dashed lines). Remarkably, on average the slow-first policy uses less time to seat all passengers than the two other policies. That the slow-first policy is superior can be intuitively explained by more synchronized seating - it better exploits the possibility to seat larger waves of passengers with similar aisle-clearing time in parallel. However, the result is nontrivial, as the similar fast-first policy is inferior to the random boarding policy for a range of congestion values $k$. Each point in the graph is an average of $10^{6}$ discrete-event runs.

deviations decreasing as the number of passengers increases $(N \rightarrow \infty)$. In the asymptotic case, analytical expressions exist for the total boarding time for both the random boarding and the slow-first (and fast-first) policies. In Fig. 3, the main parameters are derived from empirical data. More than half of the passengers are in the slow group $(p=0.55$, those with hand luggage) and their aisle-clearing time is about three times longer than for those in the fast group $(C=0.3)$. The congestion is $k=4$.

The random boarding policy treats all passengers as one group with effective aisle-clearing time $\tau_{A} \equiv \tau_{X_{A}}$, and the boarding time depends linearly on $\tau_{A} . \tau_{A}$ is determined by the distribution of the aisle-clearing times $X_{A}$, but analytical expressions for $\tau_{A}$ are generally not available. The distribution of $X_{A}$ is a mixture of the aisle-clearing time distributions of the slow and the fast groups, with respective weights $p, 1-p$ and effective aisle-clearing times $\tau_{S}, \tau_{F}$. We call the mixture concave if

$$
\tau_{A}^{2} \geqslant p \tau_{S}^{2}+(1-p) \tau_{F}^{2} \equiv \hat{\tau}_{A, \operatorname{mix}}^{2}
$$

No distributions resulting in nonconcave mixtures have, so far, been detected. However, $\tau_{A}$ is only slightly larger than $\hat{\tau}_{A \text {,mix }}$ when we examine empirical aisle-clearing time distributions.

Figure 2 presents relative boarding times for one specific choice of the parameters $p$ and $C$. Under variations in these parameters, the policies can also be compared using the relative difference

$$
D(k, p, C, N)=\frac{\left\langle T_{\mathrm{RA}}\right\rangle-\left\langle T_{\mathrm{SF}}\right\rangle}{\left\langle T_{\mathrm{SF}}\right\rangle}
$$

between the average boarding times of random boarding (RA) and slow first (SF). When we assume that $\tau_{A}=\hat{\tau}_{A \text {, mix }}$ in Eq. (1), the contour plot in Fig. 4(a) shows that $D>0$ in the $(p, C)$ unit square for $k=4$ and $N \rightarrow \infty$. Since $\left\langle T_{\mathrm{RA}}\right\rangle$ is scaled by $\tau_{A}$, it follows that $D>0$ also holds for $\tau_{A}>\hat{\tau}_{A \text {, mix }}$ (all concave mixtures).

Our main result can be stated as follows.

Theorem 1. The expected boarding time $\langle T\rangle$ is shorter for the slow-first policy than for the random boarding policy for all values of $k>0$ and $p, C \in(0,1)$, in the asymptotic regime when $N \rightarrow \infty$, if and only if the aisle-clearing time mixture is concave.

The discrete-event simulation results in Fig. 4(b) indicate that this universal result also holds for realistic numbers of passengers $N$. For the empirical aisle-clearing time distributions resulting from separating passengers into slow and fast groups based on hand luggage, the average boarding time is $8 \%$ shorter with slow first compared to random boarding when $N=180$.

We also show that the relative difference $D$ between random boarding and slow first can be maximized for fixed $k$ and $\tau_{A}=\hat{\tau}_{A \text {,mix }}$ in the asymptotic regime when $N \rightarrow \infty$. For $k>\ln (2)$ the maximum is obtained for $p \rightarrow 0$ and $C=$ $k p / \sqrt{e^{k}-k+1}$ :

$$
\sup _{p, C} D \geqslant \frac{k-\ln (2)+1}{\sqrt{k}}-1 \stackrel{k \rightarrow \infty}{\sim} \sqrt{k}-1 .
$$

For $k=4, \sup D=115 \%$ when $\tau_{A}=\hat{\tau}_{A \text {,mix }}$. For concave mixtures where $\tau_{A}>\hat{\tau}_{A \text {, mix }}$, the maximal relative difference is even higher. For large $k$, it is obviously unbounded; however, for fixed $p, C$ the asymptotic relative difference approaches a limiting value when $k \rightarrow \infty$. Proofs for Theorem 1 and Eq. (2) appear in Appendix B.

\section{SPACETIME GEOMETRY AND BOARDING TIME ASYMPTOTICS}

In this section, we give a brief introduction to how the boarding time can be approximated by the weight of the heaviest chain as $N \rightarrow \infty$. The reader is referred to Ref. [9] for a simple introduction to the analogy between airplane boarding and spacetime geometry and to Ref. [2] for a more rigorous mathematical description.

\section{A. Main parameters}

The following parameters govern the boarding process:

(i) The total number of passengers $N$. For simplicity, we assume a full airplane, i.e., that $N$ equals the number of seats in the airplane. In Fig. $1, N=8$.

(ii) The congestion $k$ is defined as the ratio of the total length of a single-file queue consisting of all passengers, and the total aisle length of the airplane. As a ratio of lengths it is a dimensionless quantity. Let $h$ be the number of seats per row, $n_{a}$ be the number of aisles, $d$ be the distance between consecutive rows and $w$ be the distance between passengers 

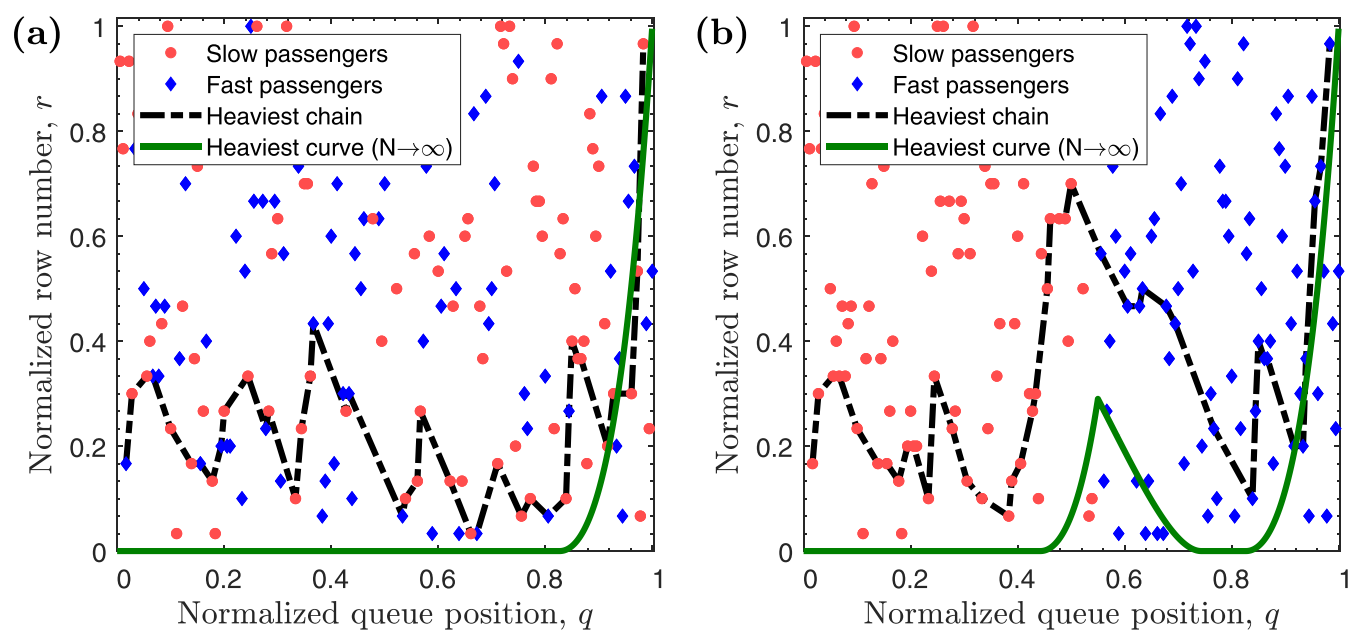

FIG. 3. $q r$ diagrams for two different boarding policies, with each of the $N=180$ passengers marked as a point, $h=6$ seats per row, and parameters $k=4, p=0.55$, and $C=0.3$ derived from empirical data. (a) Random boarding policy with all passengers in one group: The passengers are uniformly distributed over the diagram. (b) Slow-first policy with two groups: The slow passengers are in the first part of the queue (red bullets). For both policies, the boarding time is the sum of the aisle-clearing times for passengers that belong to the heaviest chain (dashed lines). A passenger cannot sit down before all preceding passengers in the chain are seated. The boarding time in each diagram is determined by the heaviest chain which follows the asymptotic limit (solid line-the geodesic), with deviations decreasing as the number of passengers increases.

as they are lined up, one after the other, in the aisle(s). Then $k=h w /\left(n_{a} d\right)$. The parameter $k$ reflects both the maximum density of passengers in the queue and the interior design of the airplane. ${ }^{1}$ In Fig. $1, k=1$ since $d=2 w, n_{a}=1$, and $h=2$.

(iii) Fraction of slow passengers $p$. In Fig. 1, the aisleclearing time of all passengers is the same, so $p=1$ or $p=0$, i.e., only a single group.

(iv) Effective aisle-clearing time $\tau_{X}$. The aisle-clearing time $X$ is the time needed for a passenger to organize bin

${ }^{1}$ The formula for calculating $k$ can be adjusted to include the relative occupancy of the airplane [33]. luggage and take a seat after reaching one's row. In Fig. 1, all passengers have an aisle-clearing time of $X \equiv 1$ time steps. The effective aisle-clearing time $\tau_{X}$ is an intrinsic parameter in the asymptotic estimate of the total boarding time and is determined by the distribution of $X$ only. Explicit expressions are generally not available, except when $X \equiv c$ is constant, in which case $\tau_{X}=c$.

(v) Ratio $C$ between the effective aisle-clearing time of the fast passengers to that of the slow passengers.

(vi) Initial queue position $q$ of a passenger normalized by the total number of passengers $N$. In Fig. 1, the sixth passenger in the queue (heading for row 2) has $q=6 / 8=0.75$.
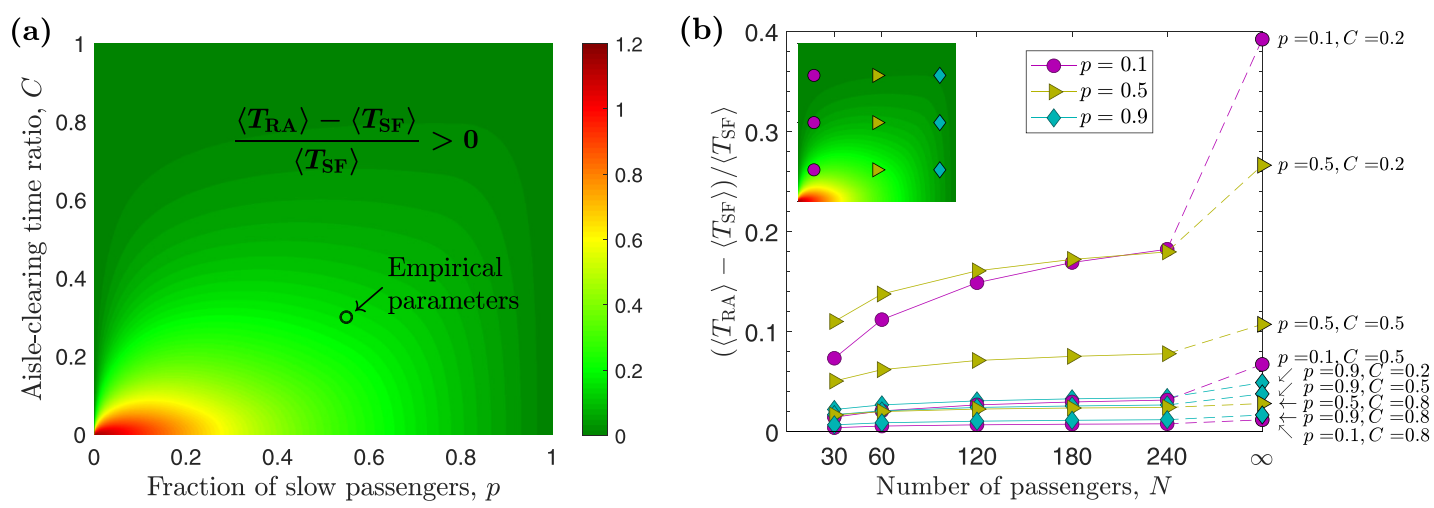

FIG. 4. Relative difference in average total boarding time $D=\left(\left\langle T_{\mathrm{RA}}\right\rangle-\left\langle T_{\mathrm{SF}}\right\rangle\right) /\left\langle T_{\mathrm{SF}}\right\rangle$ between random boarding and the slow-first policy when $k=4$. (a) The number of passengers $N \rightarrow \infty$, and we assume $\tau_{A}=\hat{\tau}_{A, \text { mix }}$. The slow-first policy gives reduced boarding time for all combinations of $p$ and $C$. The maximal relative difference of $115 \%$ is obtained for small $p$ and $C=k p / \sqrt{e^{k}-k+1}$. For the empirical parameters resulting from separating passengers into slow and fast groups based on hand luggage, the relative difference is $13 \%$ (black circle). (b) Simulation results for finite numbers of passenger $N$ confirm that random boarding is inferior to slow first. The connected points show that $D=\left(\left\langle T_{\mathrm{RA}}\right\rangle-\left\langle T_{\mathrm{SF}}\right\rangle\right) /\left\langle T_{\mathrm{SF}}\right\rangle>0$ for increasing $N$ for all combinations of parameter values $p \in\{0.1,0.5,0.9\}$ and $C \in\{0.2,0.5,0.8\}$. The rightmost points are based on the asymptotic values taken from the indicated positions in the inset contour plot from panel (a), and corrected by precise estimates of $\tau_{A}$. There are six seats per row, and the accuracy is \pm 0.0002 (as a result of $10^{6}$ runs for each finite- $N$ data point). 
(vii) Designated row number $r$ for a passenger normalized by the total number of rows. The sixth passenger in the queue in Fig. 1 has $r=2 / 4=0.5$.

In Fig. 1(b), the actual queue and row numbers are given on the axes, while the normalized $(q, r)$ values will be used in the remaining part of the paper.

We assume that only one door at the front of the airplane is used. In our numerical simulations, we use a single aisle for convenience, i.e., $n_{a}=1$ and a realistic number of $N=180$ passengers for that scenario as the default. Some simulations also report on other numbers of passengers. Moreover, the aisle at a row has to be cleared before another passenger can start clearing the aisle at the same row. However, we treat passengers as individuals and not as groups traveling together, and thus it very seldom happens that two persons heading for the same row arrive at the same time.

\section{B. Boarding policies and heaviest blocking chains}

A boarding policy defines how the passenger queue is organized before entering the airplane. Under the random boarding policy, the passengers enter the queue in random order. The $q r$ diagram in Fig. 3(a) presents a typical random boarding scenario. The points that are uniformly distributed over the unit square, represent each of the $N=180$ passengers. A scenario with the slow-first policy is shown in Fig. 3(b). The passengers are divided into two groups based on aisle-clearing time, and those who are considered slow constitute the first part of the queue. Within each group, the passengers are randomly distributed in the queue and so are the designated row numbers.

The task of finding the total boarding time can be found by identifying the groups of passengers (wave fronts) that sit down simultaneously as shown in Fig. 1 where all passengers have equal aisle-clearing time. In this case, the boarding time can be found by counting the number of such wave fronts and multiplying by the aisle-clearing time.

An important additional feature that can be observed in Fig. 1 is that each passenger in a wave front has been blocked from sitting down any earlier by a passenger in the preceding wave front. This feature is particularly useful when the aisle-clearing times vary between passengers. Instead of laboriously counting wave fronts, the blocking hierarchy between passengers is exploited. We say that a passenger $A$ blocks passenger $B$ if $A$ must clear the aisle before $B$ can take a seat. A blocking chain consists of passengers that consecutively block each other. The weight of a blocking chain is the sum of aisle-clearing times for passengers that belong to that chain, and the crucial observation is that the boarding time equals the weight of the heaviest chain.

The heaviest chain can be constructed for a given queue, by starting with a passenger $B$ in the last wave front. Passenger $B$ could be blocked by several passengers in the preceding wave fronts. The one that is closest in the queue just before $B$ arrives at $B$ 's designated row is chosen as the next passenger in the chain. A heaviest chain is obtained by proceeding in this manner, until ending up at a passenger near the front of the queue, who was never blocked. Examples of heaviest chains are shown in Figs. 1(b) and 3 for $N=8$ and 180, respectively. A much more efficient way of constructing the heaviest blocking chain is given in Ref. [20] for $k=0$, and we apply this to compute $\tau_{X}$ in Sec. V.

\section{Space-time geometry, causal curves, and curve weight}

Figure 3 indicates that the heaviest chains are close to what is called a heaviest curve. When the number of passengers $N \rightarrow \infty$, tools from causal set theory and space-time geometry can be used to show that the heaviest chains do approach the heaviest curve in the limit [2].

In space-time geometry, a Lorentzian metric defines whether there is a causal relation between events (timelike separation), and the proper times between such events are measured by causal curves with maximal length under that metric (geodesics). In airplane boarding, passengers correspond to events and the property that passenger $A$ blocks passenger $B$ corresponds to that of $A$ potentially having a causal influence on $B$. The time between seating of two passengers corresponds to the proper time between events, and when the number of events (passengers) $N \rightarrow \infty$, the time difference can be calculated by a particular Lorentzian metric.

The total boarding time is given by the blocking chain with a maximal sum of aisle-clearing times, and this heaviest blocking chain approaches a heaviest causal curve under the metric when $N \rightarrow \infty$. If we assume that the aisle-clearing time of each passenger is deterministically given by the passengers' normalized queue and row position, $X=\tau(q, r)$, the curve weight of a causal curve $r(q)$ can then be defined by [2]

$$
W(r)=\int_{q_{0}}^{q_{1}} \tau(q, r(q)) \sqrt{r^{\prime}(q)+k[1-r(q)]} d q .
$$

The square root in the integral measures the number of passengers along the causal curve, and this is weighted by the respective aisle-clearing time $\tau(q, r(q))$ along the same part of the curve. The curve is causal when the square root is real, and since the normalized queue and row number $(q, r)$ of all passengers are within the unit square, the heaviest curve must obey the same restriction. Other features of the heaviest curve are that it connects the points $(0,0)$ and $(1,1)$, it is continuous, and it is differentiable whenever $\tau(q, r)$ is continuous [2].

As noted in Sec. III, when the aisle-clearing times $X$ are stochastic, $\tau(q, r)$ in Eq. (3) can be replaced by an effective aisle-clearing time $\tau_{X}(q, r)$, which is independent of $k$ [8]. Moreover, we only consider boarding policies where each section of the queue (each passenger group) has the same aisle-clearing time distribution, such that $\tau_{X}(q, r)=\tau_{X}(q)$ takes constant values on a finite number of $q$ intervals.

\section{Asymptotic boarding time}

A general formula of Myrheim [12] states that the total boarding time converges to a multiple of the weight [Eq. (3)] of the heaviest causal curve $r$ from $(0,0)$ to $(1,1)$ within the unit square [2],

$$
\frac{T}{\sqrt{N}} \stackrel{\text { a.s. }}{\rightarrow} 2 \max _{r} W(r) .
$$

From this, the asymptotic average boarding time is given by

$$
\langle T\rangle \sim 2 \sqrt{N} \max _{r} W(r) \equiv \hat{T} .
$$


The asymptotic boarding time $\hat{T}$ is a leading term and tends to overestimate the finite- $N$ average boarding time $\langle T\rangle$ by a relative error of order $O\left(N^{-\frac{1}{3}}\right)$ for $k \leqslant \ln (2)$ and $o\left(N^{-\frac{1}{4}}\right)$ for $k>\ln (2)$ [6]. Still, the relative ranking of boarding policies is usually maintained for small $N$.

For the simple case with $k=0$ and aisle-clearing time $X \equiv 1$, Eq. (4) reduces to the Vershik-Kerov theorem which states that the number of passengers in a maximal blocking chain is approximately $2 \sqrt{N}$ for large $N$ [34]. Extending to the case where all passengers have stochastic aisle-clearing times $X$ from the same distribution, $\langle T\rangle \sim 2 \tau_{X} \sqrt{N}$ [2]. These relations are used to estimate and establish bounds for $\tau_{X}$ in Sec. V.

The result in Eq. (4) is used in Sec. VI to derive analytical expressions for the expected boarding time for both the random boarding and the slow-first boarding policies.

\section{EFFECTIVE AISLE-CLEARING TIME AND EMPIRICAL DATA}

The effective aisle-clearing time $\tau_{X}(q)$, replaces $\tau(q, r)$ in Eq. (3) when each section of the queue consists of groups of passengers with the same aisle-clearing time distribution. $\tau_{X}$ for each group depends solely on the distribution of $X$ and is independent of $k$. Explicit analytical expressions for $\tau_{X}$ are to our knowledge only known for deterministic $X \equiv c$, in which case $\tau_{X}=c$. However, the analytical bounds and extensive numerical calculations in this section indicate that $\sqrt{\left\langle X^{2}\right\rangle}$ is a relatively tight lower bound for $\tau_{X}$. The stronger conjecture, that a mixture of two distributions is concave [Eq. (1)], is supported by simulations based on empirical aisle-clearing time data.

\section{A. Lower and upper bounds on the effective aisle-clearing time}

Bounds for the effective aisle-clearing time $\tau_{X}$ appear in Ref. [8]. Let the aisle-clearing times $X$ follow a distribution with density function $f(x)$ and support in the interval $[a, b]$. Then the following inequalities hold:

$$
\frac{1}{e \sqrt{1+\ln (b / a)}} \leqslant \frac{\tau_{X}}{\sqrt{\left\langle X^{2}\right\rangle}} \leqslant e \sqrt{1+\ln (b / a)} .
$$

These bounds justify the use of $\sqrt{\left\langle X^{2}\right\rangle}$ as an estimate for $\tau_{X}$. Moreover, they show that the ratio of upper and lower bounds of $\tau_{X}$ for any distribution with support in $[a, b]$ is at most $e^{2}[\ln (b / a)+1]$, and therefore reasonably good. Here we improve those bounds and show that the estimate $\sqrt{\left\langle X^{2}\right\rangle}$ has the same bounds.

Theorem 2. Let the aisle-clearing times $X$ follow a distribution with density function $f(x)$ and support in the interval $[a, b]$. Then the following inequalities hold:

$$
\max _{u \in[a, b]} \frac{\int_{u}^{b} t f(t) d t}{\sqrt{\int_{u}^{b} f(t) d t}} \leqslant \tau_{X} \leqslant \min _{\left\{u_{i}\right\}} \sum_{i=1}^{n} u_{i} \sqrt{p_{i}} \equiv \tau_{X}^{U},
$$

where $\left\{u_{i}\right\}$ is a subdivision of $[a, b]$ with $a=u_{0}<u_{1}<u_{2}<$ $\cdots<u_{n}=b$ and $p_{i}=\operatorname{Pr}\left(u_{i-1}<X \leqslant u_{i}\right)$. The upper and lower bounds for $\tau_{X}$ also holds for $\sqrt{\left\langle X^{2}\right\rangle}$.
The proof is presented in Appendix B 5. Based on the extensive numerical calculations in Sec. V B below, we conjecture that $\tau_{X}^{2} \geqslant\left\langle X^{2}\right\rangle$ for all distributions of $X$. This is necessary for all mixtures to be concave, which in turn is a prerequisite for Theorem 1 to hold.

For a two-valued distribution taking the values $X=\tau_{S}$ and $X=\tau_{F}$ with probabilities $p$ and $1-p$, respectively, the ratio of the upper bound in Eq. (6) and the conjectured lower bound is

$$
\frac{\tau_{X}^{U}}{\sqrt{\left\langle X^{2}\right\rangle}}=\sqrt{1+\frac{2 C \sqrt{p(1-p)}}{p+C^{2}(1-p)}} .
$$

This ratio attains the maximum value $\sqrt{2}$ when $p=C^{2} /(1+$ $C^{2}$ ) for all $C \in(0,1)$.

\section{B. Numerical estimation of $\tau_{X}$ for two-valued distributions}

In this section, we base the numerical estimation of the effective aisle-clearing time $\tau_{X}$ on the asymptotic behavior of the normalized boarding time when $k=0$,

$$
\frac{\langle T\rangle}{\sqrt{N}} \sim 2 \tau_{X}-a_{1} N^{-\frac{1}{3}}
$$

where the constant $a_{1}>0$ [1].

The slow convergence towards $\tau_{X}$ in Eq. (8) requires large $N$ to obtain precise estimates of $\tau_{X}$. However, the computation of $\langle T\rangle$ is challenging when the number of passengers $N$ is large, in particular if a discrete event simulation approach is used to compute $T$ for every realization of a queue of passengers.

We describe a more efficient procedure for the estimation of $\tau_{X}$. In particular, we apply an efficient algorithm for the computation of the boarding time $T$ for a given queue. In a more general setting, this is the same as computing the heaviest increasing subsequence in a permutation (HIS) [20] which can be done in time of order $N \ln N$. The procedure is described in more detail in Appendix A. For example, we estimated the boarding time $T$ in 9 min running time for $N$ as large as $2.62 \times 10^{8}$. For comparison, a discrete event simulation would be of order $N^{3 / 2}$ in the simplest case, where all passengers are equally fast. Indeed, in this case the boarding proceeds in $\sim \sqrt{N}$ time steps, and it is necessary to update positions of $\sim N$ passengers in one step.

For two-valued distributions of the aisle-clearing time $X$ and fixed $p$, the estimates of $\tau_{X} / \sqrt{\left\langle X^{2}\right\rangle}$ are presented in Table I. The fact that no values are (significantly) below 1 seems to indicate that $\sqrt{\left\langle X^{2}\right\rangle}$ might be a lower bound for $\tau_{X}$. Hence, the two-valued mixtures in the table can with reasonable certainty be said to be concave.

For each fixed $C$, the maximum of $\tau_{X} / \sqrt{\left\langle X^{2}\right\rangle}$ in each column in Table I is obtained when $p \approx C^{2} /\left(1+C^{2}\right)$, the same relation that gives the maximum for the upper bound in Eq. (7). For the smallest value $C=0.01, \tau_{X}$ is $26 \%$ larger than $\sqrt{\left\langle X^{2}\right\rangle}$, which is relatively close to the upper limit of $41 \%$ in Eq. (7). For $C \rightarrow 1$, the distribution of $X$ approaches a constant, which implies that $\tau_{X} \rightarrow \sqrt{\left\langle X^{2}\right\rangle}$. Hence, the ratio in the table approaches 1 , which makes the upper bound artificially high for values of $C$ in this range. 
TABLE I. $\tau_{X} / \sqrt{\left\langle X^{2}\right\rangle}$ for various combinations of $C \equiv \tau_{F} / \tau_{S}$ (numbers in the top row) and $p$ (numbers in the left column) for a two-valued distribution. The precise estimates of the effective aisle-clearing time parameters $\tau_{X}$ have been obtained by extensive simulations.

\begin{tabular}{|c|c|c|c|c|c|c|c|}
\hline$p \backslash C$ & 0.01 & 0.03 & 0.1 & 0.3 & 0.5 & 0.7 & 0.9 \\
\hline 0.00001 & $1.1240(20)$ & $1.02662(52)$ & $1.00127(17)$ & & & & \\
\hline 0.00003 & $1.2049(21)$ & $1.05731(58)$ & $1.00401(20)$ & $1.00000(16)$ & & & \\
\hline 0.0001 & $1.2643(23)$ & $1.11353(79)$ & $1.01211(19)$ & $1.00038(14)$ & $0.99999(14)$ & & \\
\hline 0.0003 & $1.2385(19)$ & $1.18219(91)$ & $1.02929(20)$ & $1.00119(11)$ & $1.00015(11)$ & $1.00014(11)$ & $0.99993(11)$ \\
\hline 0.001 & $1.1646(14)$ & $1.2252(11)$ & $1.06751(38)$ & $1.00365(17)$ & $1.00025(16)$ & $0.99995(16)$ & $0.99994(16)$ \\
\hline 0.003 & $1.1028(11)$ & $1.20197(80)$ & $1.12042(45)$ & $1.01041(18)$ & $1.00155(16)$ & $1.00073(16)$ & $1.00042(16)$ \\
\hline 0.01 & $1.05402(69)$ & $1.13153(59)$ & $1.15914(47)$ & $1.02803(20)$ & $1.00462(17)$ & $1.00171(16)$ & $1.00054(16)$ \\
\hline 0.03 & $1.02896(51)$ & $1.07480(43)$ & $1.14159(42)$ & $1.05433(23)$ & $1.01187(17)$ & $1.00496(16)$ & $1.00153(16)$ \\
\hline 0.1 & $1.01313(33)$ & $1.03446(29)$ & $1.08511(32)$ & $1.07420(23)$ & $1.02711(18)$ & $1.01215(16)$ & $1.00389(16)$ \\
\hline 0.3 & $1.00455(23)$ & $1.01376(21)$ & $1.03753(23)$ & $1.05582(21)$ & $1.03520(18)$ & $1.02031(17)$ & $1.00646(16)$ \\
\hline 0.5 & $1.00232(20)$ & $1.00715(17)$ & $1.02042(20)$ & $1.03617(19)$ & $1.02858(17)$ & $1.01999(16)$ & $1.00735(16)$ \\
\hline 0.7 & $1.00142(17)$ & $1.00374(15)$ & $1.01057(18)$ & $1.02050(17)$ & $1.01899(17)$ & $1.01443(16)$ & $1.00582(16)$ \\
\hline 0.9 & $1.00051(17)$ & $1.00092(14)$ & $1.00334(16)$ & $1.00710(16)$ & $1.00779(16)$ & $1.00637(16)$ & $1.00265(16)$ \\
\hline 0.97 & $1.000043(54)$ & $1.00022(14)$ & $1.00081(16)$ & $1.00209(16)$ & $1.00257(16)$ & $1.00194(16)$ & $1.00100(16)$ \\
\hline
\end{tabular}

\section{C. $\tau_{X}$ for slow and fast groups based on empirical aisle-clearing time data}

Passengers can be separated into slow and fast groups in several ways depending on what information is known for each passenger. Based on the empirical data of Ref. [35], we apply a strategy for separating slow and fast passengers into two groups based on the number of items each passenger carries: For example, a passenger is in the slow group if he carries more than $i$ items, where $i$ is a chosen value. In the empirical data of Ref. [35], the number of hand luggage items carried by each passenger proved to be the parameter which influenced aisle-clearing time the most.

The parameters resulting from this type of luggage-based separation strategy are presented in Table II, and details of the computation are found in Appendix A. Note that the given mixtures are concave for each type of separation. This means that the main result in Theorem 1 applies (for $N \rightarrow \infty$ ).

\section{ANALYSIS OF THE RANDOM BOARDING AND SLOW-FIRST POLICIES}

We now turn to computing the asymptotic average boarding time in Eq. (4) for $k>0$, with $p, C \equiv \tau_{F} / \tau_{S}$ both in the range $(0,1)$. We show that the slow-first policy is more efficient than the random boarding policy in the entire $(k, p, C)$-parameter space in the large- $N$ limit $(N \rightarrow \infty)$ when the aisle-clearing time mixture is concave. Comparisons to simulation results for finite $N$ are also made.

\section{A. Analysis of the random boarding policy}

The random boarding policy treats all passengers as one group such that all passengers have the same aisle-clearing time distribution and effective aisle-clearing time. Then $\tau(q, r)$ can be replaced by $\tau_{A}$ in Eq. (3) and $W(r)=\tau_{A} L(r)$, where

$$
L(r)=\int_{q_{0}}^{q_{1}} \sqrt{r^{\prime}(q)+k[1-r(q)]} d q .
$$

The variational method can be used to maximize the curve length $L(r)$, and this leads to general solutions of the form $r^{*}(q)=a e^{2 k q}+b e^{k q}+1$ when $k>0$.

The constants $a, b$ are determined using the values at the start and end points: $r^{*}(0)=0$ and $r^{*}(1)=1$. A typical shape is shown in Fig. 5(a) for $k \leqslant \ln (2)$.

However, when $k>\ln (2), r^{*}(q)$ will extend below the $(q, r)$-unit square. Since the curve should be within the unit

TABLE II. Effective aisle-clearing times for groups resulting from different strategies for separating passengers into slow and fast groups. The random boarding policy with all passengers in one group has exact value $\tau_{A}=0.443$, while $\sqrt{\left\langle X_{A}^{2}\right\rangle}=0.397$ (both rounded to three significant digits). All mixtures are concave since $\tau_{A}^{2} \geqslant p \tau_{S}^{2}+(1-p) \tau_{F}^{2} \equiv \hat{\tau}_{A \text {, mix }}^{2}$. Note that in all cases $\tau_{S} \geqslant \sqrt{\left\langle X_{S}^{2}\right\rangle}$ and $\tau_{F} \geqslant \sqrt{\left\langle X_{F}^{2}\right\rangle}$.

\begin{tabular}{lcccccc}
\hline \hline Slow group & $p$ & $\sqrt{\left\langle X_{S}^{2}\right\rangle}$ & $\tau_{S}$ & $\sqrt{\left\langle X_{F}^{2}\right\rangle}$ & $\tau_{F}$ & $\hat{\tau}_{A, \text { mix }}$ \\
\hline All passengers & 1 & 0.397 & $0.44320(12)$ & & & \\
$\geqslant 1$ item & 0.55 & 0.516 & $0.56756(13)$ & 0.153 & $0.169853(43)$ & 0.437 \\
$\geqslant 2$ items & 0.15 & 0.766 & $0.82599(16)$ & 0.285 & $0.317433(83)$ & 0.435 \\
$\geqslant 3$ items & 0.04 & 1.054 & $1.12911(21)$ & 0.348 & $0.383802(94)$ & 0.435 \\
0 or $\geqslant 2$ items & 0.60 & 0.408 & $0.45560(13)$ & 0.381 & $0.41827(10)$ & 0.441 \\
2 items & 0.11 & 0.645 & $0.68426(12)$ & 0.352 & $0.39959(12)$ & 0.442 \\
1 or 2 items & 0.52 & 0.453 & $0.49250(10)$ & 0.327 & $0.37614(13)$ & 0.384 \\
1 or $\geqslant 3$ items & 0.44 & 0.476 & $0.53154(14)$ & 0.322 & $0.356265(92)$ & 0.440 \\
\hline \hline
\end{tabular}



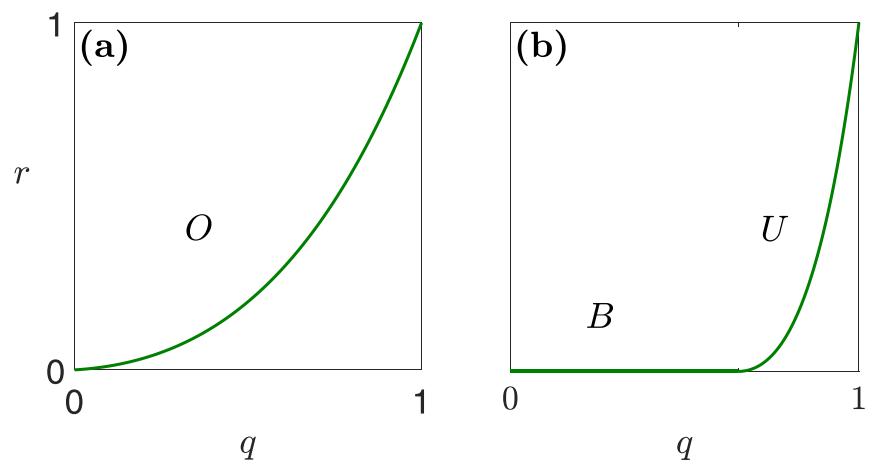

FIG. 5. The shape of the longest curve for random boarding can be either ordinary or piecewise. (a) Ordinary-type curve $(O)$ when $0<k \leqslant \ln (2)$. (b) Piecewise curve consisting of a constant function along the base $(B)$ which is smoothly continued by an upward-going ordinary-type curve $(U)$ when $k>\ln (2)$.

square, a piecewise curve as in Fig. 5(b) emerges as the longest curve (see Ref. [9] for details).

The lengths of these longest curves are computed by Eq. (9), and by Eq. (4), the expected boarding time with random boarding and effective aisle clearing time $\tau_{A}$ is to first order given by

$$
\hat{T}_{\mathrm{RA}}= \begin{cases}2 \tau_{A} \sqrt{\frac{N}{k}} \sqrt{e^{k}-1} & 0<k \leqslant \ln (2) \\ 2 \tau_{A} \sqrt{\frac{N}{k}}[k-\ln (2)+1] & \ln (2)<k .\end{cases}
$$

In Fig. 6, comparisons of the asymptotic boarding time for the random boarding policy with simulation results for $N \leqslant 240$ show that the asymptotic result in Eq. (10) tends to overestimate the boarding time. Still, the relative ranking between different parameter settings is maintained. $\tau_{A}$ is here estimated for two-valued distributions by extensive discrete-

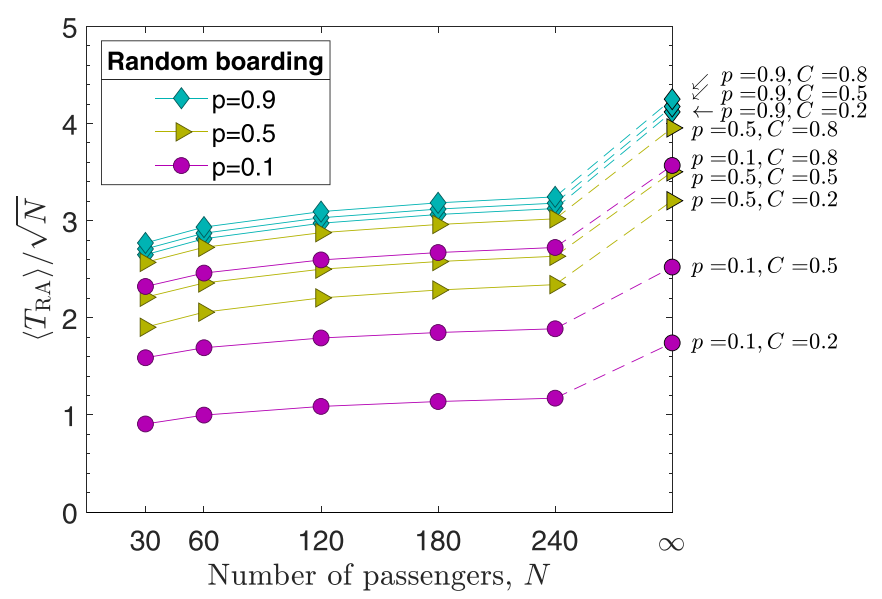

FIG. 6. Average boarding time estimates for the random boarding policy for different $(p, C)$-parameter settings. Simulation results for increasing number of passengers are compared to the asymptotic results for all combinations of parameter values $p \in\{0.1,0.5,0.9\}$ and $C \in\{0.2,0.5,0.8\} . k=4, \tau_{S}=1$, and the accuracy is \pm 0.002 (as a result of $10^{6}$ runs for each finite- $N$ data point). The rightmost points are the asymptotic values in Eq. (10). Corresponding results are presented in Ref. [9] for the slow-first and fast-first policies. event simulations to a high degree of accuracy as in Table I in Sec. V B.

\section{B. Analysis of the slow-first policy}

When there are two groups, as in, e.g., the slow-first policy, the curve weight for a function $r(q)$ on the interval $q \in(0,1)$ is given by

$$
\begin{aligned}
W_{\mathrm{SF}}(r)= & \int_{0}^{p} \tau_{S} \sqrt{r^{\prime}(q)+k(1-r(q))} d q \\
& +\int_{p}^{1} \tau_{F} \sqrt{r^{\prime}(q)+k(1-r(q))} d q \\
= & \tau_{S} L_{S}(r)+\tau_{F} L_{F}(r),
\end{aligned}
$$

where $L_{S}, L_{F}$ are curve lengths as defined in Eq. (9) and $\tau_{S}, \tau_{F}$ are the effective aisle-clearing time for the slow and fast groups, respectively.

The maximal weight $W_{\mathrm{SF}}^{*}$ of $W_{\mathrm{SF}}$ is given by the curve that gives the maximal curve weight in Eq. (11). The shape of the heaviest curve is determined by the values of $k, p, C$, where $C \equiv \tau_{F} / \tau_{S} \in(0,1)$. Detailed computations of the heaviest curves and their respective curve weights are presented in Ref. [9]. The expression for the maximal curve weight $W_{\mathrm{SF}}^{*}(k, p, C)$ is also given in Appendix B and consists of four different subfunctions. Figure 7 illustrates how the subdomains of each of the subfunctions depend on the parameters. For example, for $k \leqslant \ln (2)$, only one of the subfunctions (SF4) is present, whereas another subfunction (SF1) dominates the parameter space when $k \rightarrow \infty$.

The maximal weight $W_{\mathrm{SF}}^{*}$ is used to calculate the corresponding asymptotic boarding time $\hat{T}_{\mathrm{SF}}$ in Eq. (4). Comparisons of the asymptotic boarding time for the slow-first policy with simulation results for $N \leqslant 240$ in Ref. [9] show that the asymptotic result in Eq. (4) tends to overestimate the boarding time, but the relative ranking between different parameter settings is maintained. Corresponding analytical expressions and results for the fast-first policy are also presented in Ref. [9].

\section{Comparison of slow-first and random boarding policies}

As stated in Theorem 1 in Sec. III, the slow-first policy outperforms the random boarding policy for all values of $k>0$ and $p, C \in(0,1)$. The results are valid for concave mixtures and the proof showing that $W_{\mathrm{RA}}^{*}-W_{\mathrm{SF}}^{*}>0$ is left for Appendix B. The results of the finite- $N$ discrete-event simulations in Figs. 2 and 4(b) indicate that the result are also valid for realistic numbers of passengers $N$.

Another feature of the result is presented in Fig. 8 where the percentage of seated passengers is plotted as a function of time for the three different policies. The same empirical parameter setting as in Fig. 3 are used. The boarding time $T$ is equal to the time when the fraction of seated passengers reaches $100 \%$. Slow first ranks first $\left(T_{\mathrm{SF}}=919\right.$ time steps), then fast first $\left(T_{\mathrm{FF}}=955\right)$, and at last random boarding $\left(T_{\mathrm{RA}}=\right.$ 1037).

The graph for the slow-first policy (SF) in Fig. 8 consists of two curve segments with different slopes. The first, less steep segment corresponds to boarding dominated by slow passengers, while the steep segment is dominated by fast 

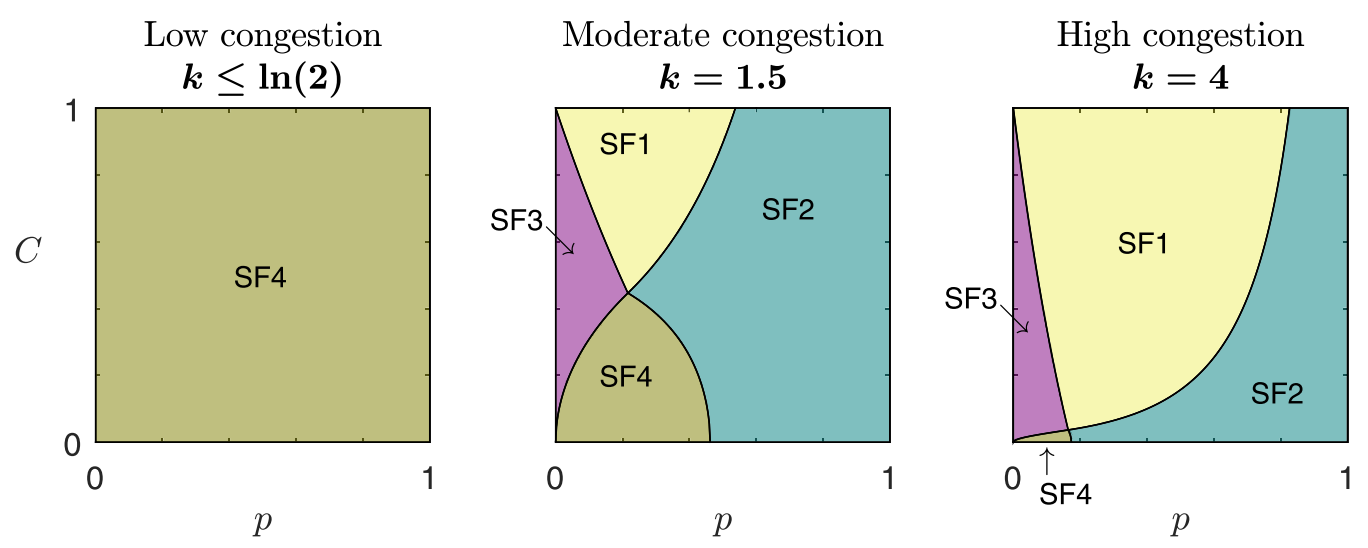

FIG. 7. The subdomains of the ( $p, C)$-unit square where the slow-first boarding time and the maximum of $W_{\mathrm{SF}}$ in Eq. (11) is represented by the different subfunctions in Eq. (B1).

passengers. As boarding starts, the queue of passengers is four times as long as the aisle $(k=4)$, and the first fast passengers in slow first enter the airplane only after a significant portion of the slow passengers are seated.

The contour plot in Fig. 4(a) indicates that the relative distance between random boarding and slow first increases for decreasing $C$. This is explicitly shown in Eqs. (B10) and (B16) for the SF4 and the SF1 regions, respectively. Ultimately, this leads to the maximal relative distance being obtained for small $C$, as stated in Eq. (2).

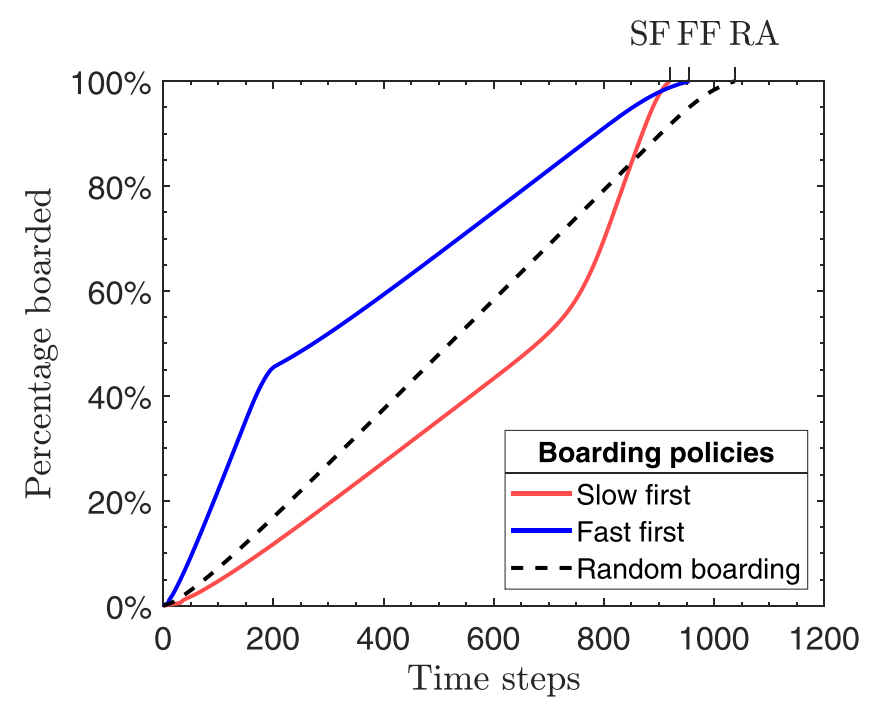

FIG. 8. Comparison of three different boarding policies. We used parameters based on the empirical data in Sec. VC, with congestion $k=4,55 \%$ slow passengers $(p=0.55)$, and assumed that the fast passengers (those without hand luggage) clear the aisle about three times faster than the slow passengers $(C=0.3)$. We assumed there are six seats per row, and a total of $N=180$ passengers. The percentage of seated passengers is plotted as a function of time. On average the slow-first policy (rightmost, red, solid line) is lagging behind all the way to around $\approx 85 \%$. However, the slow-first policy eventually seats all passengers in a shorter time span, relative to the two other policies. Fast first is second (FF; $+4 \%$ ), and the random boarding policy is third $(\mathrm{RA} ;+13 \%)$. The graph is an average of 100000 discrete-event runs.
As indicated in Fig. 7, $W_{\mathrm{SF}}^{*}=W_{\mathrm{SF} 1}^{*}$ for large $k$ when $p, C$ are fixed. In Appendix B 6, we show that

$$
\frac{W_{\mathrm{RA}}^{*}}{W_{\mathrm{SF}}^{*}} \stackrel{k \rightarrow \infty}{\longrightarrow} \frac{\sqrt{p+C^{2}(1-p)}}{p+C(1-p)}>1 .
$$

This explains why the relative difference between slow first and random boarding in Fig. 2 approaches a limiting value when $N, k \rightarrow \infty$. That both the slow-first and the fast-first policies approach the same limiting value is shown in Ref. [9]. The limiting value in Eq. (12) increases for smaller $C$, and for fixed $C$, Eq. (12) is maximized by $p=C /(1-C)$, giving the maximum $(1+C) /(2 \sqrt{C})$.

\section{Comparison of fast-first and random boarding policies}

As opposed to the slow-first policy, fast first can be both better and worse than random boarding, depending on the congestion $k$ as shown in Fig. 2 for $p=0.1, C=0.5$. The same feature is shown for other $(p, C)$ values in Fig. 9 when $k=1$ is fixed. Blue-shaded areas in the figure indicate that the asymptotic boarding time of random boarding is shorter than for the fast-first policy (negative relative difference).

In Fig. 9(a) the effective aisle-clearing time $\tau_{A}$ of random boarding is approximated by $\hat{\tau}_{A, \text { mix }}$ in the estimates, and the results suggest that fast first can be inferior to random boarding for $N=\infty$. In Fig. 9(b) this is verified for several combinations of $p, C$ values when precise estimates of $\tau_{A}$ are applied.

Figure 10 demonstrates that fast first is inferior to random boarding also for finite numbers of passengers $N$ for the particular set of parameters $k=1, p=0.1, C=0.5$ (black circles in Fig. 9). For large $N$, the relative difference approaches $-1.77 \%$, which corresponds to the minimum value in Fig. 9(b) (close to the peak value of the blue dashed curve in Fig. 2).

\section{E. Optimal separation strategies based on empirical data}

The asymptotic boarding times for random, slow-first (SF), and fast-first $(\mathrm{FF})$ boarding based on the $(p, C)$ values for some of the different separation strategies in Sec. VC are compared in Fig. 11. The congestion parameter is set to $k=4$ (typical empirical value) and we assume that $\tau_{A}=\hat{\tau}_{A \text {, mix }}$. The 

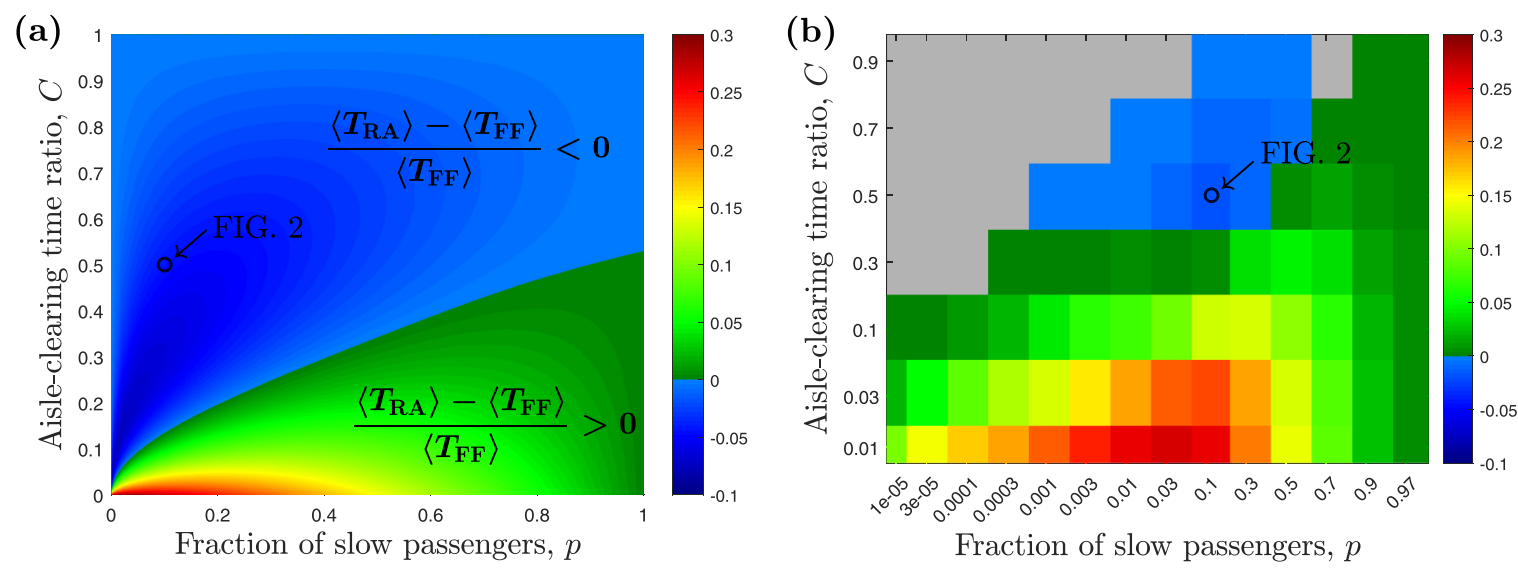

FIG. 9. Relative difference in asymptotic average boarding time $\left(\left\langle T_{\mathrm{RA}}\right\rangle-\left\langle T_{\mathrm{FF}}\right\rangle\right) /\left\langle T_{\mathrm{FF}}\right\rangle$ between the random boarding and the fast-first policies when $k=1$ as in Fig. 2. (a) The effective aisle-clearing time of random boarding $\tau_{A}$ is approximated by $\hat{\tau}_{A, \text { mix }} \equiv \sqrt{p \tau_{S}^{2}+(1-p) \tau_{F}^{2}}$, and fast first is seemingly inferior to random boarding on large portions of the ( $p, C)$ parameter space. (b) The precise estimates of $\tau_{A}$ in Table $\mathrm{I}$ are applied, and hence the asymptotic total boarding time of random boarding is larger than in panel (a). Still, fast first is inferior to random boarding for several values of $p$ small and $C$ large. Gray areas indicate that the ranking of the two policies was indeterminate within the range of \pm 2 standard deviations of the $\tau_{A}$ estimate.

inset from Fig. 4(a) shows that the $(p, C)$ values (bullets) are in the vicinity of the region with largest improvement for the slow-first policy relative to random boarding. The asymptotic boarding times of slow first and fast first relative to random boarding are shown as red and blue bullets, respectively.

The improvement by choosing the slow-first policy (and for most parameter settings also fast first) instead of random boarding is significant. From Fig. 11, one sees that when the slow and fast groups are separated based on the number of luggage items, the maximum improvement with slow first compared to random boarding is obtained when the slow group consists of those who carry luggage items. Application of the precise estimates of $\tau_{A}$ in Table II for empirical distributions (instead of assuming $\tau_{A}=\hat{\tau}_{A, \text { mix }}$ ), gives a slight improvement increase from $13 \%$ to $14 \%$. For finite number of

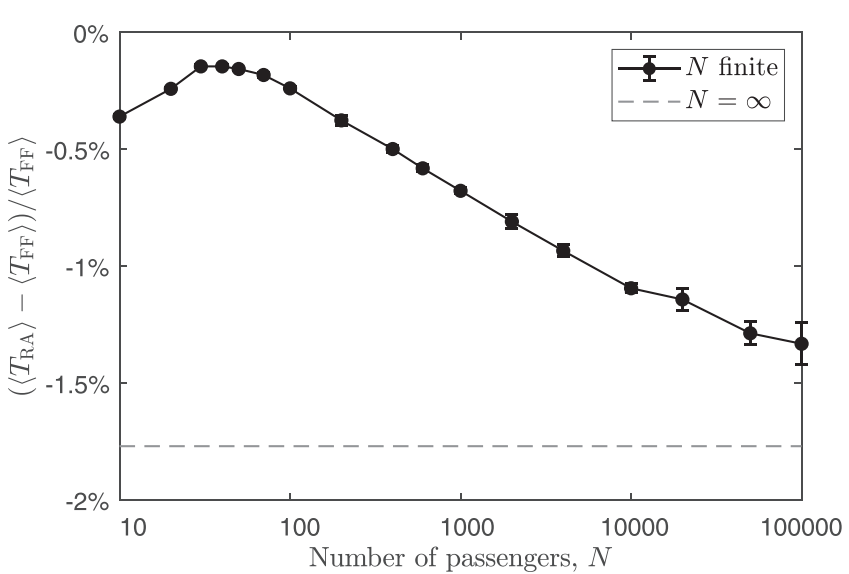

FIG. 10. Relative difference in asymptotic average boarding time $\left(\left\langle T_{\mathrm{RA}}\right\rangle-\left\langle T_{\mathrm{FF}}\right\rangle\right) /\left\langle T_{\mathrm{FF}}\right\rangle$ between the random boarding and the fast-first policies when $(k, p, C)=(1,0.1,0.5)$. For this setting, the graph indicates that random boarding is superior also for finite $N$. For large $N$, the relative difference approaches $-1.77 \%$, which is the asymptotic value $(N \rightarrow \infty)$ marked with a black circle in Fig. 9(b). passengers $N=180$ and empirical aisle-clearing time distributions, discrete event simulations give that the improvement of slow first compared to random boarding is $8 \%$ when the slow passengers are those who carry luggage items.

When the slow and fast groups are separated (clairvoyantly) based on those $p=30 \%$ who have the slowest aisle-clearing times, the maximum improvement with slow first compared to random boarding is $21 \%$ in Fig. 11. Note, however, that this separation strategy is merely theoretical, as the exact aisle-clearing times are not known before seating is completed. The choice of quantile $p$ that is used as a threshold to separate slow from fast passengers is quite robust to variations as the improvement is quite similar for all values of $p$ in the range $p \in[0.2,0.4]$.

\section{DISCUSSION AND OUTLOOK}

In this paper, we recast the airplane boarding problem to the setting of Lorentzian geometry and show that the slow-first boarding policy is superior to random boarding for any set of parameters when the number of passengers $N \rightarrow \infty$. The analytical result is presented in Theorem 1 and hinges on a concavity assumption regarding the effective aisle-clearing time of groups of passengers with varying aisle-clearing times. This parameter is not available in closed form, but extensive numerical computations enable us to validate the concavity assumption with a high degree of certainty.

As stated in Eq. (2), the maximal relative distance between random boarding and slow first is obtained for a small ratio $C$ between effective aisle-clearing times of the fast and slow groups. This seems to be an important feature that distinguishes the slow-first policy from random boarding. $C$ being small implies that the aisle-clearing time of the slow passengers is much longer than for the fast passengers. In random boarding, a few slow passengers will then be able to block and delay many fast passengers from getting to their seats, in particular when the congestion $k$ is large. With the slow-first policy, the variability within each group is reduced, and this 


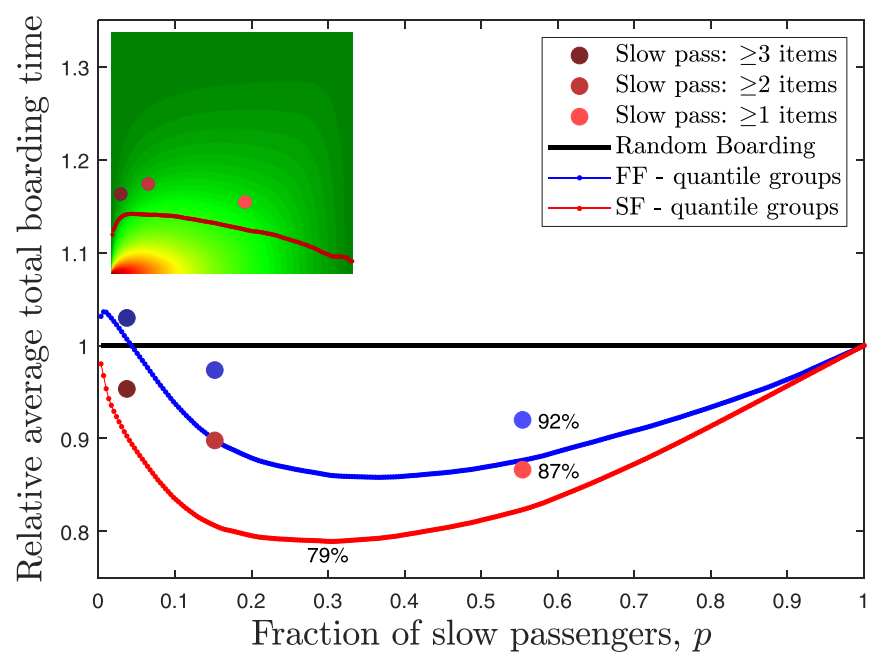

FIG. 11. Relative asymptotic boarding time for slow first (SF, red) and fast first (FF, blue) compared to random boarding for different $(p, C)$ values based on empirical data and $k=4$. Results for luggage-based separation strategies are shown as red and blue bullets, while separations that are based on quantiles of the empirical aisleclearing time distribution are shown as dot-lined curves. Red-colored results are asymptotic values taken from the indicated positions in the inset contour plot of the relative difference between random boarding and slow first from Fig. 4(a). The effective aisle-clearing time of random boarding $\tau_{A}$ is approximated by $\hat{\tau}_{A, \text { mix }} \equiv \sqrt{p \tau_{S}^{2}+(1-p) \tau_{F}^{2}}$ (conjectured as a lower bound). If the slow group are those with 1 luggage item or more, the improvement by choosing slow first instead of random boarding is $13 \%$. The corresponding result for fast first is $8 \%$. However, separation where slow passengers have three or more luggage items results in a fast-first policy which could be inferior to random boarding. The improvement can be up to $21 \%$ when slow and fast passengers are separated according to quantiles in the aisle-clearing time distribution.

enables more synchronized clearing of the aisle and less effect of local interference between passengers. Since the relative difference tends to increase with $C$ small and $k$ large, local interference seems to be the crucial detrimental aspect of random boarding. This aspect seems to be much less pronounced within the more homogeneous groups in the slow-first policy.

While slow first is always superior to random boarding, the ranking of fast first and random boarding depends on the values of the parameters $(k, p, C)$. Fast first enables synchronized seating in the same way as slow first, and the positive effect of this is higher when $C$ is small and $k$ is large. However, random boarding is superior for large $C$ and small $k$. A heuristic explanation for the inconsistent ranking of the two policies could be found in Fig. 8. The graphs indicate that the rate of seating is slower in the start phase for all boarding policies. While the slow-first policy enables parallel seating in the transition phase between the groups [9], there is in effect two starting phases with the fast-first policy-one for the fast group and one for the slow group. For certain parameter settings (e.g., large $C$ and small $p$ ), the positive effect of improved local synchronization in fast first is not enough to offset this.

The present paper together with Ref. [9] provides a complete picture of the relation between three policies which are actually practiced by airlines-random boarding, fast first, and slow first - across all parameter settings. In ongoing work with several additional coauthors, we are extending this work in new directions, both in terms of optimization functions and policies, in terms of finding optimal policies and considering more than two groups.

Considering more than two groups, a possible extension of the slow-first policy, could be to distribute the passengers into more groups with even less variation within each group. Since slow first is better, the groups should be sequenced in the queue according to descending effective aisle-clearing times. In fact, preliminary results indicate that this strategy reduces the total boarding time and ultimately a queue where all passengers are ranked according to descending aisle-clearing time is even better.

With respect to optimization functions, one could consider average individual boarding time (customer experience) rather than total boarding time. Then the ranking of the three policies slow first, random boarding, and fast first is reversed, and the performance differences between fast first and slow first are more pronounced compared to total boarding time. Consequently, the total boarding time of slow first is shorter, but the average passenger suffers longer waits in the queue. This leads to the introduction of new policies which interpolate between slow first and fast first and provide the benefits of both.

The problem of finding optimal queue-row placements is also of great interest. Such placements form space-time lenses with respect to proper time for positive mass particles, in the sense that $(0,0)$ and $(1,1)$ become conjugate points. This leads to some efficient lens constructions, but as boarding policies they are often very difficult to implement. However, for large values of $k$, there are near-optimal lenses with small amounts of aberrations that correspond to reasonable boarding policies.

\section{ACKNOWLEDGMENTS}

The work of Eitan Bachmat was supported by the Deutsche Forschungsgemeinschaft (DFG) through the grant 'Airplane Boarding' (Grant No. JA 2311/3-1). The authors acknowledge the use of resources provided by the Latvian Grid Infrastructure and High Performance Computing Centre of Riga Technical University.

\section{APPENDIX A: PROCEDURE FOR ESTIMATION OF $\tau_{X}$}

In this Appendix, we describe an efficient procedure for the estimation of the effective aisle-clearing time $\tau_{X}$, when the aisle-clearing time $X$ of all passengers in a group follow a common distribution. Since $\tau_{X}$ is independent of $k$, we refer to the case when $k=0$.

First, we establish an efficient algorithm for the computation of the boarding time $T$ for a given queue, by recasting the problem to the computation of a heaviest increasing subsequence (HIS). We use a variant of the algorithm in Ref. [20]. Then $\langle T\rangle$ is estimated by simulation for a series of $N$ values. The first-order approximation $\langle T\rangle /(2 \sqrt{N}) \rightarrow \tau_{X}$ (see Sec. IV D) is improved by an extrapolation to $N \rightarrow \infty$ using the extended large- $N$ behavior in Eq. (8).

We first considered an example where the weights $\tau_{F}=$ 1 (fast passengers) and $\tau_{S}=2$ (slow passengers) are assigned randomly, each with probability $p=1 / 2$ (the fraction 
TABLE III. The dependence on $N$ of the normalized boarding time $\langle T\rangle / \sqrt{N}$ of random boarding when $p=0.5=C$.

\begin{tabular}{ll}
\hline \hline$N$ & $\langle T\rangle / \sqrt{N}$ \\
\hline 1000 & $2.9802(14)$ \\
8000 & $3.11190(75)$ \\
64000 & $3.1798(12)$ \\
512000 & $3.21753(61)$ \\
$\infty$ & $3.2553(17)$ \\
\hline \hline
\end{tabular}

of slow passengers). We tested the algorithm for $N=$ $1000,8000,64000$, and 512000 passengers. A total of 10000 simulation runs were performed for $N \leqslant 8000$ and 1000 simulation runs for $N \geqslant 64000$.

The estimated average boarding times $\langle T\rangle$ for random boarding (the values of HIS) normalized to $\sqrt{N}$ are given in Table III. The asymptotic value at $N=\infty$ is obtained by an extrapolation (see the following section), taking into account that corrections to scaling can be expanded in powers of $N^{-1 / 3}$ as in Eq. (8).

The normalized asymptotic boarding time for both slow first and fast first is just $2 \sqrt{\left\langle X^{2}\right\rangle} \approx 3.1622777$ (when $k=$ $0, p=0.5, C=0.5)$. It is seen that, in this particular case, the random boarding policy is a bit worse (slower).

\section{1. $\tau_{X}$ for two-valued distributions}

In order to estimate the unknown constant $\tau_{X}$ and to investigate whether it is bounded from below by $\sqrt{\left\langle X^{2}\right\rangle}$, we take the asymptotic result in Eq. (8) and define the ratio

$$
\phi_{N} \equiv \frac{T / \sqrt{N}}{2 \sqrt{\left\langle X^{2}\right\rangle}} \sim \phi_{\infty}-\tilde{a}_{1} N^{-\frac{1}{3}},
$$

where $\phi_{\infty} \equiv \tau_{X} / \sqrt{\left\langle X^{2}\right\rangle}$. Then the following extrapolation algorithm to estimate $\phi_{\infty}$ has been used.

We performed simulations at a hierarchy of $N=N_{i}$ values: $N_{0}, N_{1}=8 N_{0}, N_{2}=8^{2} N_{0}$, etc. This resulted in a corresponding hierarchy of $\phi_{N}$ values, i.e., $\phi_{N_{0}}, \phi_{N_{1}}, \phi_{N_{2}}$, etc. Using that $\phi_{N}$ in Eq. (A1) is linear in $N^{-1 / 3}$, we obtain a sequence of estimates for $\phi_{\infty}$ by linear extrapolation, i.e.,

$$
\phi_{i}^{\text {lin }}=2 \phi_{N_{i}}-\phi_{N_{i-1}}
$$

for $i \geqslant 1$. With the ansatz that Eq. (A1) can be expanded further in powers of $N^{-\frac{1}{3}}$, we also considered the quadratic extrapolation

$$
\phi_{i}^{\text {quad }}=\frac{1}{3}\left(8 \phi_{N_{i}}-6 \phi_{N_{i-1}}+\phi_{N_{i-2}}\right) .
$$

Each next value is expected to be twice closer to the asymptotic value at $N \rightarrow \infty$ for the original sequence $\phi_{N_{i}}$, four times closer for $\phi_{i}^{\text {lin }}$, and eight times closer for $\phi_{i}^{\text {quad }}$. We also observed that at a given number $M$ of simulation runs, each next value in any of these sequences has statistical error (standard deviation $\sigma$ ) smaller by approximately 2 .

We used the following criterion: The maximal number of points (passengers) $N$ has to be large enough to ensure that the systematical extrapolation error is much smaller than $\sigma$. The
TABLE IV. The values of $\tau_{X, N}$ ( $\tau_{A}$ for all passengers, $\tau_{F}$ for fast passengers, and $\tau_{S}$ for slow passengers when the slow passengers are those carrying hand luggage), extracted from 10000 runs of HIS simulations depending on the number of passengers $N$. The asymptotic estimates are obtained by a linear and a quadratic extrapolation in the variable $N^{-1 / 3}$.

\begin{tabular}{llll}
\hline \hline$N$ & \multicolumn{1}{c}{$\tau_{A}$} & \multicolumn{1}{c}{$\tau_{F}$} & \multicolumn{1}{c}{$\tau_{S}$} \\
\hline 100 & $0.33337(62)$ & $0.12969(22)$ & $0.44204(70)$ \\
800 & $0.38168(35)$ & $0.14771(12)$ & $0.49844(39)$ \\
6400 & $0.41048(18)$ & $0.158080(65)$ & $0.53049(20)$ \\
51200 & $0.426061(93)$ & $0.163696(33)$ & $0.54820(10)$ \\
409600 & $0.434405(48)$ & $0.166659(17)$ & $0.557788(53)$ \\
3276800 & $0.438745(24)$ & $0.1682272(86)$ & $0.562650(27)$ \\
$\infty$ (linear extr.) & $0.443086(68)$ & $0.169795(24)$ & $0.567511(75)$ \\
$\infty$ (quadr. extr.) & $0.44320(12)$ & $0.169853(43)$ & $0.56756(13)$ \\
\hline \hline
\end{tabular}

statistical error for $\phi_{i}^{\text {lin }}$ is somewhat smaller than that of $\phi_{i}^{\text {quad }}$; however, in some of the considered cases, the above criterion was not well satisfied for $\phi_{i}^{\text {lin }}$ but it was satisfied for $\phi_{i}^{\text {quad }}$. Therefore, $\phi_{i}^{\text {quad }}$ values for the largest $i$ have been used as the final asymptotic estimates of $\phi_{\infty}$.

Calculations within the range of parameters $0.01 \leqslant C \leqslant$ 0.9 and $0.00001 \leqslant p \leqslant 0.97$ have been performed. The simulation parameter $N_{0}$ ranged from 10 to 1000 (larger for smaller $C$ and $p$ ), whereas the maximal $i$ has been fixed equal to 7 . The number of $\mathrm{MC}$ realizations (simulation runs) ranged from 10000 (for $N_{0}=10$ ) to 500 (for $N_{0}=1000$ ). The results are collected in Table I.

The most accurate value has been obtained for $p=0.97$ and $C=0.01$. In this case, the original less accurate value $0.99981(16)$ was smaller by $\approx 1.2 \sigma$ than unity, and therefore we performed extra simulations to verify whether or not it is $<1$. As a result, the possibility that $\phi_{\infty}<1$ holds in this case has not been confirmed. In all other cases $\phi_{\infty}$ is either $>1$ or the deviation below unity is within $1 \sigma$.

Thus, our simulation and calculation results do not allow us to conclude that $\tau_{X}$ in some cases is smaller than $\sqrt{\left\langle X^{2}\right\rangle}$ for two-valued distributions.

\section{2. $\tau_{X}$ for multivalued distributions based on empirical data}

We consider the aisle-clearing time distribution data in Ref. [35]. The mean boarding time $\langle T\rangle$ for the multivalue distribution is determined by the HIS algorithm where each weight is taken from the same distribution. For each distribution, we define a hierarchy of $\tau_{X, N}$ values where for each $N$, $\tau_{X, N}=\langle T\rangle /(2 \sqrt{N})$. As for the two-valued distributions, we use linear and quadratic polynomials of $N^{-1 / 3}$ [corresponding to Eqs. (A2) and (A3)] for an extrapolation to $N=\infty$, based on the idea that $\langle T\rangle / \sqrt{N}$ can be expanded in powers of $N^{-1 / 3}$. The results for 10000 simulation runs are collected in Table IV for the separation strategy when the slow passengers are those carrying hand luggage.

A fast convergence of a series of extrapolated values shows that such an extrapolation is accurate enough. The values of the linear extrapolation in Table IV have smaller statistical errors, but systematical extrapolation errors are smaller and 
practically negligible for the values of the quadratic extrapolation. Hence, $\tau_{A}=0.44320(12), \tau_{F}=0.169853$ (43), and $\tau_{S}=0.56756(13)$ can be assumed as the final estimates. The estimates for $\tau_{F}$ and $\tau_{S}$ in Table II when the passengers are divided into fast and slow groups in other ways are obtained in a similar manner.

\section{APPENDIX B: PROOFS}

There are five different combinations of RA and SF subfunctions which we treat in the subsections below in order to show that $W_{\mathrm{RA}}^{*}-W_{\mathrm{SF}}^{*}>0$ [for $k \leqslant \ln (2)$ there is one combination, and for $k>\ln (2)$ there are four combinations]:

$$
W_{\mathrm{RA}}^{*}= \begin{cases}\tau_{A} \sqrt{\frac{1}{k}} \sqrt{e^{k}-1} & 0<k \leqslant \ln (2) \\ \tau_{A} \sqrt{\frac{1}{k}}[k-\ln (2)+1] & \ln (2)<k .\end{cases}
$$

We assume that

$$
\tau_{A}^{2}=p \tau_{S}^{2}+(1-p) \tau_{F}^{2}=\tau_{S}^{2}\left[p+C^{2}(1-p)\right] \equiv \hat{\tau}_{A, \operatorname{mix}}^{2},
$$

and prove that $W_{\mathrm{RA}}^{*}-W_{\mathrm{SF}}^{*}>0$. Obviously, this inequality will also hold if $\tau_{A}>\hat{\tau}_{A \text {, mix }}$.

From Ref. [9], we have that $W_{\mathrm{SF}}^{*}$ can be represented by one of four different subfunctions,

$$
\begin{array}{rlrl}
W_{\mathrm{SF} 1}^{*} & =\frac{\tau_{S}}{\sqrt{k}}\left[k p(1-C)+k C+1+C \ln \left(\frac{C}{1+C}\right)-\ln \left(\frac{2}{1+C}\right)\right] & \max \left\{C_{2}, C_{1}\right\} \leqslant C, \\
W_{\mathrm{SF} 2}^{*}= & \frac{\tau_{S}}{\sqrt{k}}\left[k p+1-\ln \left(\frac{2}{1+C^{2}\left(e^{k(1-p)}-1\right)}\right)\right] & C_{3}^{2} \leqslant C^{2} \leqslant C_{1}^{2}, \\
W_{\mathrm{SF} 3}^{*}=\frac{\tau_{S}}{\sqrt{k}} C\left[k+1-\ln (2)+\frac{\sqrt{\left(e^{k p}-1\right)\left(1-C^{2}\right)}}{C}-\ln \left(1+\frac{\sqrt{\left(e^{k p}-1\right)\left(1-C^{2}\right)}}{C}\right)\right] & C_{4}^{2} \leqslant C^{2} \leqslant C_{2}^{2}, \\
W_{\mathrm{SF} 4}^{*}=\frac{\tau_{S}}{\sqrt{k}} \sqrt{\left(e^{k p}-1\right)+C^{2}\left(e^{k}-e^{k p}\right)} & C^{2} \leqslant \min \left\{C_{3}^{2}, C_{4}^{2}\right\} .
\end{array}
$$

Here $C \equiv \tau_{F} / \tau_{S} \in(0,1)$, and the borders between the different subdomains as shown in Fig. 7 are given by

$$
\begin{array}{ll}
\text { SF1-SF2: } & C_{1} \equiv\left(e^{k(1-p)}-1\right)^{-1}, \\
\text { SF1-SF3: } & C_{2} \equiv 2 e^{-k p}-1, \\
\text { SF2-SF4: } & C_{3}^{2} \equiv\left(2-e^{k p}\right) /\left(e^{k}-e^{k p}\right), \\
\text { SF3-SF4: } & C_{4}^{2} \equiv 4\left(e^{k p}-1\right) /\left[e^{2 k}-4\left(e^{k}-e^{k p}\right)\right] .
\end{array}
$$

For $k<\ln (2)$, both $C_{3}>1, C_{4}>1$, and then $W_{\mathrm{SF}}^{*}=W_{\mathrm{SF} 4}^{*}$. For $k \geqslant \ln (2)$, all subfunctions are present. The borders meet at a vortex point where $p=p^{*} \equiv \ln \left[2 /\left(1+C^{*}\right)\right] / k$ and $C=C^{*} \equiv 2 e^{-k}$.

\section{Proof of Theorem 1: $W_{\mathrm{RA}}^{*}-W_{\mathrm{SF}}^{*}>0$ when $k \leqslant \ln (2)$}

We show that the difference of the squares is decreasing in $C$ for all $p, k$. It is straightforward to show that the difference is 0 both for $C=1, p=0$, and $p=1$. Since the difference is 0 for $C=1$, the difference is positive for $C<1$ :

$$
\begin{aligned}
\frac{k}{\tau_{S}^{2}}\left(W_{\mathrm{RA}}^{* 2}-W_{\mathrm{SF} 4}^{* 2}\right) & =\left[p+C^{2}(1-p)\right]\left(e^{k}-1\right)-\left(e^{k p}-1\right)-C^{2}\left(e^{k}-e^{k p}\right), \\
\frac{k}{\tau_{S}^{2}} \frac{\partial\left(W_{\mathrm{RA}}^{* 2}-W_{\mathrm{SF} 4}^{* 2}\right)}{\partial\left(C^{2}\right)} & =(1-p)\left(e^{k}-1\right)-\left(e^{k}-e^{k p}\right) \equiv g(k, p), \\
\frac{\partial g}{\partial p} & =-\left(e^{k}-1\right)+k e^{k p}, \\
\frac{\partial^{2} g}{\partial p^{2}} & =k^{2} e^{k p}>0 .
\end{aligned}
$$

Since $g(k, 0)=0=g(k, 1), g(k, p)<0$ for all $p \in(0,1)$. There is also negative curvature in the $p$ direction:

$$
\begin{gathered}
\frac{k}{\tau_{S}^{2}} \frac{\partial\left(W_{\mathrm{RA}}^{* 2}-W_{\mathrm{SF} 4}^{* 2}\right)}{\partial p}=\left(1-C^{2}\right)\left(e^{k}-1-k e^{k p}\right), \\
\frac{k}{\tau_{S}^{2}} \frac{\partial^{2}\left(W_{\mathrm{RA}}^{* 2}-W_{\mathrm{SF} 4}^{* 2}\right)}{\partial p^{2}}=-\left(1-C^{2}\right) k^{2} e^{k p}<0 .
\end{gathered}
$$




\section{Proof of Theorem 1: Necessity of concave mixture}

The necessity of concavity of the aisle-clearing time mixture is shown by a counterexample for small $k$. In Eq. (B3), it is assumed that $\tau_{A}^{2}=\hat{\tau}_{A \text {, mix }}^{2} \equiv p \tau_{S}^{2}+(1-p) \tau_{F}^{2}$. Instead, assume that the mixture is nonconcave, i.e., for $\varepsilon>0$, let $\tau_{A}^{2}=$ $(1-\varepsilon) \hat{\tau}_{A, \text { mix }}^{2}<\hat{\tau}_{A, \text { mix }}^{2}$. This gives

$$
\frac{1}{\tau_{S}^{2}}\left(W_{\mathrm{RA}}^{* 2}-W_{\mathrm{SF} 4}^{* 2}\right)=-\varepsilon \hat{\tau}_{A, \text { mix }}^{2}+O(k),
$$

which means that the boarding time will be less for random boarding than for slow first for sufficiently small $k$.

\section{Proof of Theorem 1: $W_{\mathrm{RA}}^{*}-W_{\mathrm{SF}}^{*}>0$ when $k>\ln (2)$}

It is straightforward to show that the difference is 0 both for $C=1, p=0$, and $p=1$.

\section{a. $R A$ vs $S F 1$}

The difference has negative curvature in $p$,

$$
\begin{aligned}
& \frac{\sqrt{k}}{\tau_{S}}\left(W_{\mathrm{RA}}^{*}-W_{\mathrm{SF} 1}^{*}\right)= \sqrt{C^{2}+p\left(1-C^{2}\right)}[k+1-\ln (2)]-k[C+p(1-C)] \\
&-1+\ln (2)-C \ln (C)-(1-C) \ln (1+C), \\
& \frac{\sqrt{k}}{\tau_{S}} \frac{\partial\left(W_{\mathrm{RA}}^{*}-W_{\mathrm{SF} 1}^{*}\right)}{\partial p}= \frac{\left(1-C^{2}\right)[k+1-\ln (2)]}{2 \sqrt{C^{2}+p\left(1-C^{2}\right)}}-k(1-C), \\
& \frac{\sqrt{k}}{\tau_{S}} \frac{\partial^{2}\left(W_{\mathrm{RA}}^{*}-W_{\mathrm{SF} 1}^{*}\right)}{\partial p^{2}}=-\frac{\left(1-C^{2}\right)^{2}[k+1-\ln (2)]}{4\left[C^{2}+p\left(1-C^{2}\right)\right]^{\frac{3}{2}}}<0 .
\end{aligned}
$$

That the difference is positive depends on non-negative difference on the SF3-SF1 and SF1-SF2 borders, respectively (see Appendixes B 3 b and B 3 c below).

\section{b. RA vs $S F 2$}

The difference has negative curvature in $p$,

$$
\begin{aligned}
& \frac{\sqrt{k}}{\tau_{S}}\left(W_{\mathrm{RA}}^{*}-W_{\mathrm{SF} 2}^{*}\right)= \sqrt{C^{2}+p\left(1-C^{2}\right)}[k+1-\ln (2)]-k p-1+\ln (2) \\
&-\ln \left[1+C^{2}\left(e^{k(1-p)}-1\right)\right], \\
& \frac{\sqrt{k}}{\tau_{S}} \frac{\partial\left(W_{\mathrm{RA}}^{*}-W_{\mathrm{SF} 4}^{*}\right)}{\partial p}= \frac{\left(1-C^{2}\right)[k+1-\ln (2)]}{2\left[C^{2}+\left(1-C^{2}\right) p\right]^{\frac{1}{2}}}-k+\frac{k C^{2} e^{k}}{C^{2} e^{k}+\left(1-C^{2}\right) e^{k p}}, \\
& \frac{\sqrt{k}}{\tau_{S}} \frac{\partial^{2}\left(W_{\mathrm{RA}}^{*}-W_{\mathrm{SF} 4}^{*}\right)}{\partial p^{2}}=-\frac{\left(1-C^{2}\right)^{2}[k+1-\ln (2)]}{4\left[C^{2}+\left(1-C^{2}\right) p\right]^{\frac{3}{2}}}-\frac{k^{2} C^{2} e^{k}\left(1-C^{2}\right) e^{k p}}{\left[C^{2} e^{k}+\left(1-C^{2}\right) e^{k p}\right]^{2}}<0 .
\end{aligned}
$$

That the difference is positive depends on non-negative differences on the SF1-SF2 (smooth) and SF4-SF2 borders (see Appendix B 3 c) and that the difference is zero for $p=1$.

\section{c. RA vs SF3}

Set $y \equiv e^{k p}-1$. Then $y \in(0,1)$, since $k p<\ln (2)$. Set $R \equiv \sqrt{\left(e^{k p}-1\right)\left(1-C^{2}\right)} / C=\sqrt{y\left(1-C^{2}\right)} / C>0$. To stay in SF3 toward the SF3-SF1 border, $y \leqslant R /(2+R)$, and toward the SF3-SF4 border $R \leqslant\left(e^{k}-2\right) / 2$. This means that $R$ is constant at the SF3-SF4 border for fixed $k$.

The reparameterization gives that

$$
\begin{aligned}
\frac{\sqrt{k}}{C \tau_{S}}\left(W_{\mathrm{RA}}^{*}-W_{\mathrm{SF} 3}^{*}\right) & =(k+L)\left(\sqrt{1+\frac{R^{2} \ln (1+y)}{k y}}-1\right)+\ln (1+R)-R \\
& \equiv f(y, R, k),
\end{aligned}
$$

where $L \equiv 1-\ln (2)$. 
We first find the values of $k \in(\ln (2), \infty)$ that minimizes $f$ in Eq. (B5) for fixed values of $A \equiv R^{2} \ln (1+y) / y$ :

$$
\frac{\partial f}{\partial k}=\frac{A(A-4 L)\left(k-\frac{L}{1-\sqrt{\frac{4 L}{A}}}\right)[\sqrt{A}(k-L)+2 k \sqrt{L}]}{4 k^{4}(\sqrt{A}+2 \sqrt{L}) \sqrt{1+\frac{A}{k}}\left[1+\frac{A}{k}\left(1-\frac{k+L}{2 k}\right)+\sqrt{1+\frac{A}{k}}\right]} .
$$

Lower bounds for $f$ on the SF3 domain are given by three different values of $k$, depending on the value of $A=A(R, y)$ :

(1) When $A \leqslant 4 L, \frac{\partial f}{\partial k}<0$, and $f$ is minimized when $k \rightarrow k_{1} \equiv \infty$.

(2) When $A>4 L, f$ is minimized when $\frac{\partial f}{\partial k}=0$, which is obtained when $k$ is set to

$$
k_{2}(A) \equiv \frac{L}{1-\sqrt{\frac{4 L}{A}}} .
$$

Note that $k_{2}(A)$ decreases toward $L$ when $A$ increases.

(3) When $A>4 L /[1-L / \ln (2)]^{2} \approx 3.95$, then $k_{2}<\ln (2)$, and $k=k_{3} \equiv \ln (2)$ gives a lower bound for $f$ since $k>\ln (2)$ by default and $\frac{\partial f}{\partial k}>0$ when $k>k_{2}$.

Since $\ln (1+y) / y$ is decreasing in $y, \partial f / \partial y<0$. Furthermore, since $y \leqslant R /(R+2)$ on the SF3 domain, lower bounds for $f$ can be found by setting $y=y_{1} \equiv R$ (used below for small $R$ ) or $y=y_{2} \equiv 1$ (used below for large $R$ ) since both $y_{1}$ and $y_{2}$ are larger than $R /(R+2)$. Given the fixed value of $R, A(R, y(R))$ determines which of the three values of $k$ above should be chosen to obtain a lower bound for $f$ :

(1) Assume $A \leqslant 4 L$, and set $k=k_{1}=\infty$ and $y=y_{1}=R$. Then $G_{1}(R) \equiv f(y=R, R, k=\infty)$ is a lower bound for $f$ on SF3 when $R<1.40$, since then $A=R \ln (1+R)<4 L$, and

$$
\begin{aligned}
& G_{1}(R)=\frac{A}{2}+\ln (1+R)-R=\frac{R}{2} \ln (1+R)+\ln (1+R)-R, \\
& G_{1}^{\prime \prime}(R)=\frac{1}{2(1+R)^{2}}>0 .
\end{aligned}
$$

Since $G_{1}(0)=G_{1}^{\prime}(0)=0$, then $G_{1}(R)>0$ when $R>0$, and in particular for $R \in(0,1.40)$.

(2) Assume $A>4 L$, and set $k=k_{2}$ and $y=y_{2}=1$. Then $A=R^{2} \ln (2)>4 L$ when $R>2 \sqrt{L / \ln (2)} \approx 1.33$. Also, with this choice of $y, k_{2}=L R /[R-2 \sqrt{L / \ln (2)}]>\ln (2)$ when $R<2 \sqrt{L \ln (2)} /[2 \ln (2)-1] \approx 2.38$. Then $G_{2}(R) \equiv f\left(y=1, R, k_{2}\right)$ is a lower bound for $f$ on SF3, at least when $R>1.35$, and

$$
\begin{aligned}
G_{2}(R) & =2 L\left(\sqrt{\frac{A}{L}}-1\right)+\ln (1+R)-R \\
& =2 L\left(R \sqrt{\frac{\ln (2)}{L}}-1\right)+\ln (1+R)-R, \\
G_{2}^{\prime \prime}(R) & =-\frac{1}{(1+R)^{2}}<0 .
\end{aligned}
$$

Since $G_{2}(1) \approx 0.0018, G_{2}(3) \approx 0.54$, then $G_{2}(R)>0$ when $R \in[1,3]$, and in particular for $R \in[1.35,3]$.

(3) Assume $A>4 L /[1-L / \ln (2)]^{2}$ (which is the case when $y=y_{2}=1$ and $R>2.38$ ), and set $k=k_{3}=\ln (2)$. Then $G_{3}(R) \equiv f\left(y=1, R, k_{3}\right)$ is a lower bound for $f$ on SF3 when $R>2.38$, and

$$
\begin{aligned}
G_{3}(R) & =\left(\sqrt{\left.1+\frac{A}{\ln (2)}-1\right)+\ln (1+R)-R}\right. \\
& =\left(\sqrt{R^{2}+1}-1\right)+\ln (1+R)-R, \\
G_{3}^{\prime}(R) & =\frac{R}{\sqrt{1+R^{2}}}-\frac{R}{1+R}>0,
\end{aligned}
$$

and since $G_{3}(0)=0$, we get that $G_{3}(R)>0$ when $R>0$, and in particular for $R>2.38$.

\section{d. RA vs SF4}

We show that the difference of the squares is decreasing in $C$ for all $p, k$,

$$
\frac{k}{\tau_{S}^{2}}\left(W_{\mathrm{RA}}^{* 2}-W_{\mathrm{SF} 4}^{* 2}\right)=\left[p+C^{2}(1-p)\right][k-\ln (2)+1]^{2}-\left(e^{k p}-1\right)-C^{2}\left(e^{k}-e^{k p}\right) \equiv f(k, p, C),
$$




$$
\begin{aligned}
\frac{k}{\tau_{S}^{2}} \frac{\partial\left(W_{\mathrm{RA}}^{* 2}-W_{\mathrm{SF} 4}^{* 2}\right)}{\partial\left(C^{2}\right)} & =(1-p)[k-\ln (2)+1]^{2}-\left(e^{k}-e^{k p}\right) \\
& \leqslant(1-p)\left(e^{k}-1\right)-\left(e^{k}-e^{k p}\right) \equiv g(k, p)<0 .
\end{aligned}
$$

The first inequality in Eq. (B7) is due to

$$
h(k) \equiv\left(e^{k}-1\right)-[k-\ln (2)+1]^{2}, \quad h^{\prime}(k)=e^{k}-2[k-\ln (2)+1], \quad h^{\prime \prime}(k)=e^{k}-2>0,
$$

when $k>\ln (2)$. Since $h(\ln (2))=0=h^{\prime}(\ln (2)), h(k)>0$ when $k>\ln (2)$. The second inequality in Eq. (B7) was shown in Appendix B 1 for all $k>0, p \in(0,1)$.

There is also negative curvature in the $p$ direction,

$$
\begin{aligned}
\frac{k}{\tau_{S}^{2}} \frac{\partial\left(W_{\mathrm{RA}}^{* 2}-W_{\mathrm{SF} 4}^{* 2}\right)}{\partial p} & =\left(1-C^{2}\right)\left\{[k+1-\ln (2)]^{2}-k e^{k p}\right\}, \\
\frac{k}{\tau_{S}^{2}} \frac{\partial^{2}\left(W_{\mathrm{RA}}^{* 2}-W_{\mathrm{SF} 4}^{* 2}\right)}{\partial p^{2}} & =-\left(1-C^{2}\right) k^{2} e^{k p}<0 .
\end{aligned}
$$

Due to the negative curvature in $p$, the difference in Eq. (B6) is positive on SF4 if it is positive on the SF3-SF4 border (proved in Appendix B 3 c) and on the SF4-SF2 border.

At the latter border $p<\ln (2) / k$ and $C<C^{*} \equiv 2 e^{-k}$. Due to the negative curvature in $p$, it is sufficient to show that the difference is positive on a line extending from $C=0$ to $C=C^{*}$ with fixed $p=\ln (2) / k$. Since the difference is decreasing in $C$ [Eq. (B7)], it is sufficient to show that the difference is positive at the point $\left(p=\ln (2) / k, C=C^{*}\right)$,

$$
f\left(k, p=\frac{\ln (2)}{k}, C=C^{*}\right)=\frac{1}{k}\left[l_{2}+4 e^{-2 k}\left(k-l_{2}\right)\right]\left[k-l_{2}+1\right]^{2}-1-4 e^{-2 k}\left(e^{k}-2\right) \equiv F(k),
$$

where $l_{2} \equiv \ln (2)$. Let $K \equiv k-l_{2}$ (such that $k>\ln (2)$ implies $K>0$ ), then

$$
\frac{k e^{2 K} F(k)}{K}=e^{2 K}\left(K l_{2}+2 l_{2}-1\right)+(K+1)^{2}-2\left(e^{K}-1\right)-2 l_{2} \frac{e^{K}-1}{K} \equiv h(K) .
$$

Since $h(0)=0$, it is sufficient to show that $h^{\prime}(K)>0$ to prove that $h(K)>0$ for $K>0$ [which in turn implies that $F(k)>0$ for $k>\ln (2)]$,

$$
\frac{h^{\prime}(K)}{2}=\underbrace{e^{2 K}\left(K l_{2}+2.5 l_{2}-1\right)+(K+1)-e^{K}}_{g_{1}(K)}-\underbrace{l_{2} \frac{e^{K}(K-1)+1}{K^{2}}}_{g_{2}(K)} .
$$

For $K \leqslant 1$, the first term $g_{1}(K)$ is increasing. Hence, a lower bound is $g_{1}(K) \geqslant g_{1}(0)=0.73$ when $K \geqslant 0$. An upper bound for $g_{2}(K)$ is found by $e^{K} \geqslant 1+K+K^{2} / 2$ and that the factor $(K-1)$ is negative for $0<K<1$. Then we can write for $0<K<1$

$$
g_{2}(K) \leqslant l_{2} \frac{\left(1+K+K^{2} / 2\right)(K-1)+1}{K^{2}}=\frac{l_{2}}{2}(1+K) \equiv \tilde{g}_{2}(K) .
$$

Using that this upper bound is increasing, $g_{2}(K) \leqslant \tilde{g}_{2}(1)=l_{2}=0.69$ when $K \leqslant 1$. This implies that $g_{1}(K)-g_{2}(K)>$ $0.73-0.69>0$ for $K \leqslant 1$. For $K>1$, it is straightforward to show that each of the negative terms in Eq. (B8) are dominated by one of the respective positive terms, completing the proof of Theorem 1.

\section{Proof of Eq. (2): Maximal relative distance between RA and SF policies}

We propose that the maximal relative distance $\left(W_{\mathrm{RA}}^{*}-W_{\mathrm{SF}}^{*}\right) / W_{\mathrm{SF}}^{*}=W_{\mathrm{RA}}^{*} / W_{\mathrm{SF}}^{*}-1$ is in the SF4 region. For fixed $k>\ln (2)$, we therefore seek the maximum of

$$
\frac{1}{[k-\ln (2)+1]^{2}}\left(\frac{W_{\mathrm{RA}}^{*}}{W_{\mathrm{SF} 4}^{*}}\right)^{2}=\frac{p+C^{2}(1-p)}{e^{k p}-1+C^{2}\left(e^{k}-e^{k p}\right)}=\frac{C^{2}+p\left(1-C^{2}\right)}{e^{k p}\left(1-C^{2}\right)+C^{2} e^{k}-1} \equiv g(k, p, C) .
$$

The same equation applies for $k \leqslant \ln (2)$, except that the leftmost denominator is exchanged with $e^{k}-1$.

Partial differentiation of $g$ gives

$$
\frac{\partial g}{\partial\left(C^{2}\right)}=\frac{e^{k p}-1-p\left(e^{k}-1\right)}{\left[e^{k p}-1+C^{2}\left(e^{k}-e^{k p}\right)\right]^{2}} \leqslant 0,
$$

since the numerator of $\partial g / \partial\left(C^{2}\right)$ has positive curvature in $p$ and equals 0 both for $p=0$ and $p=1$. Consequently, for fixed $k, p$ and $C \in(0,1), g$ is maximized when $C \rightarrow 0$. 
Partial differentiation of $g$ with respect to $p$ gives

$$
\frac{1}{1-C^{2}} \frac{\partial g}{\partial p}=\frac{e^{k p}(1-k p)-1+C^{2}\left[e^{k}-e^{k p}(k-k p+1)\right]}{\left[e^{k p}-1+C^{2}\left(e^{k}-e^{k p}\right)\right]^{2}} .
$$

A local optimum if found by setting Eq. (B11) to zero. This gives

$$
\begin{aligned}
C^{2} & =\frac{1-e^{k p}(1-k p)}{e^{k}-e^{k p}[k(1-p)+1]} \\
\stackrel{p \rightarrow 0}{\sim} & \frac{(k p)^{2}}{e^{k}-k+1} \stackrel{k \rightarrow \infty}{\sim} \frac{(k p)^{2}}{e^{k}} .
\end{aligned}
$$

For fixed $k$, the numerator in Eq. (B12) is increasing from 0 when $p>0$, and the denominator is non-negative and bounded from above. Hence, $p$ must be small to minimize $C$.

The asymptotic optimal value for $C^{2}$ in Eq. (B13) can be inserted into Eq. (B9), which gives the maximum

$$
\begin{aligned}
\left(\frac{W_{\mathrm{RA}}^{*}}{W_{\mathrm{SF} 4}^{*}}\right)^{2}= & {[k-\ln (2)+1]^{2} \frac{p+\frac{(k p)^{2}}{e^{k}-k+1}(1-p)}{e^{k p}-1+\frac{(k p)^{2}}{e^{k}-k+1}\left(e^{k}-e^{k p}\right)} } \\
& \stackrel{p \rightarrow 0}{\sim} \frac{[k-\ln (2)+1]^{2}}{k} \frac{\left(e^{k}-k+1\right)+k^{2} p}{\left(e^{k}-k+1\right)+k p\left(e^{k}-1\right)} \\
& \stackrel{k \rightarrow \infty}{\sim} k .
\end{aligned}
$$

This means that for fixed $k$, the maximal relative difference between random boarding and slow first equals $[k-\ln (2)+1] / \sqrt{k}-$ 1 and is obtained for $p$ small and $C \approx k p / \sqrt{e^{k}-k+1}$.

For large $k$, the maximal relative difference between random boarding and slow first is obtained for $p$ small and $C \approx k p / \sqrt{e^{k}}$, and by increasing $k$ the relative difference can be infinitely large,

$$
\frac{W_{\mathrm{RA}}^{*}-W_{\mathrm{SF} 4}^{*}}{W_{\mathrm{SF} 4}^{*}} \approx \sqrt{k}-1 .
$$

Theoretically, there could be other local maxima in other subdomains. However, numerical inspections indicate that the given solution is the global maximum.

\section{Proof of Theorem 2: Bounds on the effective aisle-clearing time $\tau_{X}$}

Since $\tau_{X}$ is independent of $k$, we look at the case $k=0$ where the weight of the heaviest chain is asymptotically $2 \tau_{X} \sqrt{N}$ for large $N$. We apply the Vershik-Kerov theorem which states that when $X$ is deterministic and $k=0$, the number of points in a longest chain is asymptotically $2 \sqrt{N}$.

For $\tau_{X}$ and a given $u \in[a, b]$, the lower bound is established by considering the weight of the longest sequence of points with weight at least $u$. There are roughly $\int_{u}^{b} f(t) d t N$ such points and so by the Vershik-Kerov theorem we have a longest chain of size roughly $2 \sqrt{\int_{u}^{b} f(t) d t} \sqrt{N}$ points. The average weight of a point in this chain is the same as the average weight of a point conditioned on being larger than $u$, i.e., $\int_{u}^{b} t f(t) d t / \int_{u}^{b} f(t) d t$. Since this holds for any $u$, the result follows.

The upper bound on $\tau_{X}$ is obtained by replacing $X$ by $X_{u}$ that takes the values $u_{i}$ with probability $p_{i}=\operatorname{Pr}\left(u_{i-1}<X \leqslant u_{i}\right)$. Obviously $\tau_{X} \leqslant \tau_{X_{u}}$, since $X_{u}$ dominates $X$. Consider the heaviest chain with respect to $X_{u}$. The number of points with weight $u_{i}$ in this chain is bounded from above by the size of longest chain of such points which by Vershik-Kerov has a total weight of roughly $2 u_{i} \sqrt{p_{i}} \sqrt{N}$. Adding this up gives that the weight of the heaviest chain is estimated from above by $2\left(\sum_{i=1}^{n} u_{i} \sqrt{p_{i}}\right) \sqrt{N}$. The upper bound in Eq. (6) is obtained since this holds for all subdivisions.

For the lower bound of $\sqrt{\left\langle X^{2}\right\rangle}$, we apply the Cauchy-Schwartz inequality $\int_{a}^{b} g(t) f(t) d t \leqslant \sqrt{\int_{a}^{b} g^{2}(t) d t} \sqrt{\int_{a}^{b} h^{2}(t) d t}$. For a given $u \in[a, b]$, let $g=1_{[u, b]} \sqrt{f(t)}$ and $h=t \sqrt{f(t)}$ (here $1_{[u, b]}$ is the indicator of the interval $[u, b]$ which equals 1 for points in the interval and 0 otherwise). For these $f$ and $g$, the two sides of the lower bound inequality coincide with the two sides of Cauchy-Schwartz.

Regarding the upper bound of $\sqrt{\left\langle X^{2}\right\rangle}$, we note that since the $\ell_{1}$ norm of a vector is greater or equal to its $\ell_{2}$ norm,

$$
\sum_{i=1}^{n} u_{i} \sqrt{p_{i}} \geqslant \sqrt{\sum_{i=1}^{n} u_{i}^{2} p_{i}}=\sqrt{\left\langle X_{u}^{2}\right\rangle} \geqslant \sqrt{\left\langle X^{2}\right\rangle} .
$$




\section{Asymptotic relative distance between RA and SF policies for $k \rightarrow \infty$}

For large $k$, the SF1 domain dominates the $p, C$ unit square which follows straightforwardly from the SF1 boundaries in Eq. (B2). For fixed $p, C$, the relative distance is independent of $k$ for large $k$ and Eq. (12) follows since

$$
\begin{gathered}
\frac{W_{\mathrm{RA}}^{*}}{W_{\mathrm{S} 1}^{*}}=\frac{\sqrt{p+C^{2}(1-p)}[k+1-\ln (2)]}{\left[p+C^{2}(1-p)\right] k+1-\ln (2)+(1-C) \ln (1+C)+C \ln (C)} \\
\stackrel{p \rightarrow \infty}{\longrightarrow} \frac{p(1-p)\left(1-C^{2}\right)}{p+C(1-p)} \equiv h(p, C)=1+\frac{\sqrt{p+C^{2}(1-p)}}{[p+C(1-p)]\left[p+C(1-p)+\sqrt{p+C^{2}(1-p)}\right]}>1
\end{gathered}
$$

The last equality comes from the identity $a / b \equiv 1+\left(a^{2}-b^{2}\right) /[b(a+b)]$. The partial derivatives of $h$ are

$$
\frac{\partial h}{\partial C}=-\frac{p(1-p)(1-C)}{2 \sqrt{p+C^{2}(1-p)}[p+C(1-p)]^{2}}<0, \quad \frac{\partial h}{\partial p}=\frac{(1-C)^{2}[C-p(1-C)]}{2 \sqrt{p+C^{2}(1-p)}[p+C(1-p)]^{2}} .
$$

This gives that the asymptotic function $h(p, C)$ in Eq. (B15) is increasing with decreasing $C$. For fixed $C$, the maximum is at $p=C /(1-C)$ with maximum value $h^{*}=(1+C) /(2 \sqrt{C})$.

For fixed $p, C$, the partial derivative with respect to $k$ gives that the relative difference in Eq. (B14) is decreasing with increasing $k$ toward the asymptotic function $h(p, C)$, if

$$
p>1+\frac{1}{1-\ln (2)}\left[\ln (1+C)+\frac{C \ln (C)}{1-C}\right] .
$$

This means that $h(p, C)$ is a lower bound for $W_{\mathrm{RA}}^{*} / W_{\mathrm{SF} 1}^{*}$ in this area of the $p, C$ unit square for all $k>\ln (2)$. On the other hand, if $p$ is smaller than the right-hand side of Eq. (B17), the relative difference is increasing toward $h(p, C)$.

[1] E. Bachmat, D. Berend, L. Sapir, S. Skiena, and N. Stolyarov, Analysis of aeroplane boarding via spacetime geometry and random matrix theory, J. Phys. A: Math. Gen. 39, L453 (2006).

[2] E. Bachmat, Airplane boarding, disk scheduling, and lorentzian geometry, in Mathematical Adventures in Performance Analysis: From Storage Systems, through Airplane Boarding, to Express Line Queues (Springer International, Cham, 2014), pp. 51-129.

[3] N. Bernstein, Comment on Time needed to board an airplane: A power law and the structure behind it, Phys. Rev. E 86, 023101 (2012).

[4] M. Brics, J. Kaupužs, and R. Mahnke, Scaling behavior of an airplane-boarding model, Phys. Rev. E 87, 042117 (2013).

[5] Y. Baek, M. Ha, and H. Jeong, Impact of sequential disorder on the scaling behavior of airplane boarding time, Phys. Rev. E 87, 052803 (2013).

[6] E. Bachmat, V. Khachaturov, and R. Kuperman, Optimal backto-front airplane boarding, Phys. Rev. E 87, 062805 (2013).

[7] R. Mahnke, J. Kaupužs, and M. Brics, Air traffic, boarding and scaling exponents, in Traffic and Granular Flow '13, edited by M. Chraibi, M. Boltes, A. Schadschneider, and A. Seyfried (Springer International, Cham, 2015), pp. 305-314.

[8] E. Bachmat, Airplane boarding meets express line queues, Eur. J. Oper. Res. 275, 1165 (2019).

[9] S. Erland, J. Kaupužs, V. Frette, R. Pugatch, and E. Bachmat, Lorentzian-geometry-based analysis of airplane boarding policies highlights "slow passengers first" as better, Phys. Rev. E 100, 062313 (2019).

[10] V. Frette and P. C. Hemmer, Time needed to board an airplane: A power law and the structure behind it, Phys. Rev. E 85, 011130 (2012).
[11] L. Bombelli, J. Lee, D. Meyer, and R. D. Sorkin, Space-Time as a Causal Set, Phys. Rev. Lett. 59, 521 (1987).

[12] J. Myrheim, Statistical geometry, CERN Report No. CERNTH-2538, 1978 (unpublished).

[13] G. t'Hooft, Quantum gravity: A fundamental problem and some radical ideas, in Recent Developments in Gravitation: Cargèse 1978, NATO Advanced Study Institutes Series (Series B: Physics) Vol. 44, edited by M. Lévy and S. Deser (Springer, Berlin, 1979), pp. 323-345.

[14] G. Brightwell and R. Gregory, Structure of Random Discrete Spacetime, Phys. Rev. Lett. 66, 260 (1991).

[15] S. Surya, Evidence for the continuum in $2 d$ causal set quantum gravity, Class. Quantum Gravity 29, 132001 (2012).

[16] D. Stroud, Generalized effective-medium approach to the conductivity of an inhomogeneous material, Phys. Rev. B 12, 3368 (1975).

[17] Z. Fan, A microstructural approach to the effective transport properties of multiphase composites, Philos. Mag. A 73, 1663 (1996).

[18] M. Wang and N. Pan, Predictions of effective physical properties of complex multiphase materials, Mater. Sci. Eng. R Rep. 63, 1 (2008).

[19] M. M. Braun and L. Pilon, Effective optical properties of nonabsorbing nanoporous thin films, Thin Solid Films 496, 505 (2006).

[20] G. Jacobson and K.-P. Vo, Heaviest increasing/common subsequence problems, in Combinatorial Pattern Matching, edited by A. Apostolico, M. Crochemore, Z. Galil, and U. Manber (Springer, Berlin, 1992), pp. 52-66.

[21] S. Neumann, Is the boarding process on the critical path of the airplane turn-around? Eur. J. Oper. Res. 277, 128 (2019). 
[22] C. Delcea, L.-A. Cotfas, M. Salari, and J. R. Milne, Investigating the random seat boarding method without seat assignments with common boarding practices using an agent-based modeling, Sustainability 10, 4623 (2018).

[23] F. Jaehn and S. Neumann, Airplane boarding, Eur. J. Oper. Res. 244, 339 (2015).

[24] H. Van Landeghem and A. Beuselinck, Reducing passenger boarding time in airplanes: A simulation based approach, Eur. J. Oper. Res. 142, 294 (2002).

[25] J. H. Steffen, Optimal boarding method for airline passengers, J. Air Transp. Manag. 14, 146 (2008).

[26] J. H. Steffen, A statistical mechanics model for free-for-all airplane passenger boarding, Am. J. Phys. 76, 1114 (2008).

[27] J. H. Steffen and J. Hotchkiss, Experimental test of airplane boarding methods, J. Air Transp. Manag. 18, 64 (2012).

[28] J. Audenaert, K. Verbeeck, and G. Berghe, Multi-agent based simulation for boarding, in Proceedings of The 21st Benelux Conference on Artificial Intelligence, BNAIC 2009, Eindhoven, the Netherlands, 2009 (Eindhoven University of Technology, Eindhoven, 2009), pp. 3-10.

[29] S.-J. Qiang, B. Jia, D.-F. Xie, and Z.-Y. Gao, Reducing airplane boarding time by accounting for passengers' individual proper- ties: A simulation based on cellular automaton, J. Air Transp. Manag. 40, 42 (2014).

[30] R. J. Milne and A. R. Kelly, A new method for boarding passengers onto an airplane, J. Air Transp. Manag. 34, 93 (2014).

[31] R. Milne and M. Salari, Optimization of assigning passengers to seats on airplanes based on their carry-on luggage, J. Air Transp. Manag. 54, 104 (2016).

[32] G. Notomista, M. Selvaggio, F. Sbrizzi, G. D. Maio, S. Grazioso, and M. Botsch, A fast airplane boarding strategy using online seat assignment based on passenger classification, J. Air Transp. Manag. 53, 140 (2016).

[33] E. Bachmat, D. Berend, L. Sapir, S. Skiena, and N. Stolyarov, Analysis of airplane boarding times, Oper. Res. 57, 499 (2009).

[34] A. M. Vershik and S. V. Kerov, Asymptotics of the Plancherel measure of the symmetric group and the limiting form of Young tables, Dokl. Akad. Nauk SSSR 233, 1024 (1977) [Sov. Math. Dokl. 18, 527 (1977)].

[35] A. Steiner and M. Philipp, Speeding up the airplane boarding process by using pre-boarding areas, in Proceedings of the 9th Swiss Transport Research Conference, Ascona (Switzerland, 2009). 